BNL-112597-2016-JA

\title{
Atomistic Interrogation of B-N Co-dopant Structures and Their Electronic Effects in Graphene
}

Theanne Schiros, Dennis Nordlund, Lucia Palova, Liuyan Zhao, Mark Levendorf, Cherno Jaye, David Reichman, Jiwoong Park, Mark Hybertsen, Abhay Pasupathy

Submitted to the Journal of ACS Nano

June 2016

Center for Functional Nanomaterials

Brookhaven National Laboratory

\author{
U.S. Department of Energy \\ USDOE Office of Science (SC), \\ Basic Energy Sciences (BES) (SC-22)
}

\footnotetext{
Notice: This manuscript has been authored by employees of Brookhaven Science Associates, LLC under Contract No. DE- SC0012704 with the U.S. Department of Energy. The publisher by accepting the manuscript for publication acknowledges that the United States Government retains a non-exclusive, paidup, irrevocable, world-wide license to publish or reproduce the published form of this manuscript, or allow others to do so, for United States Government purposes.
} 


\section{DISCLAIMER}

This report was prepared as an account of work sponsored by an agency of the United States Government. Neither the United States Government nor any agency thereof, nor any of their employees, nor any of their contractors, subcontractors, or their employees, makes any warranty, express or implied, or assumes any legal liability or responsibility for the accuracy, completeness, or any third party's use or the results of such use of any information, apparatus, product, or process disclosed, or represents that its use would not infringe privately owned rights. Reference herein to any specific commercial product, process, or service by trade name, trademark, manufacturer, or otherwise, does not necessarily constitute or imply its endorsement, recommendation, or favoring by the United States Government or any agency thereof or its contractors or subcontractors. The views and opinions of authors expressed herein do not necessarily state or reflect those of the United States Government or any agency thereof. 


\title{
Atomistic Interrogation Of B-N Co-Dopant
}

\section{Structures And Their Electronic Effects In Graphene}

\author{
AUTHOR NAMES
}

Theanne Schiros ${ }^{\text {la,b* }}$, Dennis Nordlund ${ }^{2}$, Lucia Palova ${ }^{3}$, Liuyan Zhao ${ }^{4}$, Mark Levendorf ${ }^{5}$, Cherno Jaye $^{6}$, David Reichman ${ }^{3}$, Jiwoong Park ${ }^{5}$, Mark Hybertsen ${ }^{7}$, Abhay Pasupathy ${ }^{4}$

\section{AUTHOR ADDRESS}

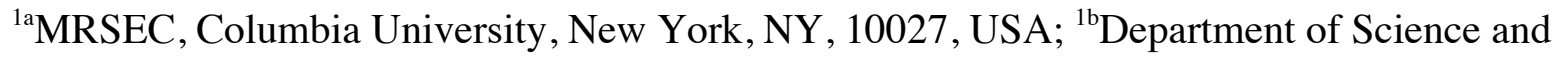
Mathematics, Fashion Institute of Technology/State University of New York, New York, NY, 10001, USA.

${ }^{2}$ Stanford Synchrotron Radiation Lightsource, SLAC National Accelerator Laboratory, Menlo Park, CA 94025, USA

${ }^{3}$ Department of Chemistry, Columbia University, New York, NY 10027, USA

${ }^{4}$ Department of Physics, Columbia University, New York, NY 10027, USA

${ }^{5}$ Chemistry Department, Cornell University, Ithaca, New York 10065, USA

${ }^{6}$ Materials Measurement Laboratory, National Institute of Standards and Technology,

Gaithersburg, Maryland 20899,USA

${ }^{7}$ Center for Functional Nanomaterials, Brookhaven National Laboratory, Upton, New York 11973, USA 
KEYWORDS. Graphene, doping, x-ray spectroscopy, scanning tunneling microscopy, chemical bonding, atomic design, electronic structure, work function.

ABSTRACT. Chemical doping has been demonstrated to be an effective method for producing high quality, large area graphene with controlled carrier concentrations and an atomically tailored work function. The emergent optoelectronic properties and surface reactivity of carbon nanostructures are dictated by the microstructure of atomic dopants. Co-doping of graphene with boron and nitrogen offers the possibility to further tune the electronic properties of graphene at the atomic level, potentially creating $\mathrm{p}$ - and n-type domains in a single carbon sheet, opening a gap between valence and conduction bands in the 2-D semi-metal, enhancing catalytic activity and altering optical properties. Using a suite of high-resolution synchrotron-based x-ray techniques, scanning tunneling microscopy (STM) and density functional theory (DFT)-based computation we visualize and characterize B-N dopant bond structures and their electronic effects at the atomic level in single layer graphene grown on a copper substrate. We find there is a thermodynamic driving force for B and $\mathrm{N}$ atoms to cluster into BNC structures in graphene, rather than randomly distribute into isolated $\mathrm{B}$ and $\mathrm{N}$ graphitic dopants, although under the present growth conditions, kinetics limit segregation of large B-N domains. We observe that the doping effect of these BNC structures, which open a small band gap in graphene, follows the B: $\mathrm{N}$ ratio $(\mathrm{B}>\mathrm{N}$, p-type; $\mathrm{B}<\mathrm{N}, \mathrm{n}$-type; $\mathrm{B}=\mathrm{N}$, neutral). We attribute this to the comparable electron withdrawing and donating effects, respectively, of individual graphitic $\mathrm{B}$ and $\mathrm{N}$ dopants, although local electrostatics also play a role in the work function change. 
Atomically thin materials, including graphene and h-BN and their hybrids, have recently been the subject of vigorous exploration with regard to both the fundamental physics of 2-D materials, ${ }^{1,2}$ interfaces, ${ }^{3}$ and heterojunctions ${ }^{4-6}$ as well as their promise for a number of device applications, including field-effect transistors, photovoltaics, ${ }^{4,7}$ fuel cells,${ }^{8,9}$ lithium ion batteries,${ }^{10}$ supercapacitors, ${ }^{11,12}$ biosensors, transparent electrodes, and high-performance photocatalysts, as discussed in ref.13 and references therein. Two-dimensional and layered materials offer atomically sharp, crystallographically well-defined interfaces free of disorder and charge traps that limit mobility, and offer ohmic-type contacts for efficient charge extraction in device applications. $^{4,5,14}$ In addition to its high transparency, conductivity, and flexibility, the technological promise of graphene is strongly enhanced by the ability to tune its electronic properties by electrostatic gating, ${ }^{15}$ surface transfer doping, ${ }^{7}$ and substitutional doping. ${ }^{16-27}$ This feature underlies the promise of graphene emerging as a universal contact in atomically tailored heterojunctions in high performance (opto)electronic devices. Chemical doping of graphene by interaction with foreign atoms ${ }^{17}$ such as nitrogen ${ }^{16,19,20-22,28,29}$ boron $^{23-25}$ and chlorine, ${ }^{26,27}$ can produce $\mathrm{n}$ - and $\mathrm{p}$-type large area graphene with high, controlled carrier concentrations and a tunable work function. Calculations suggest that, depending on the degree and type of correlation in the dopant sites, a band gap may be opened..$^{29,30}$ Co-doping graphene with $\mathrm{B}$ and $\mathrm{N},{ }^{8,31}$ which respectively have electron withdrawing and donating effects on the carbon host ${ }^{21,22,25}$ presents the opportunity to atomically engineer conductive films with p- and n-type domains. Co-doping may open a gap between valence and conduction bands ${ }^{32-36}$ a requirement for transistor-based devices, and enhance the performance of carbon-based materials in electrochemical energy conversion and storage applications. ${ }^{7-12}$ A recent density functional theory (DFT) study also predicts that, in contrast to individual $\mathrm{B}$ or $\mathrm{N}$ doping, $\mathrm{B}-\mathrm{N}$ co-doping can uniquely tailor the 
optical properties of graphene in the visible region. ${ }^{37}$ The electronic structure of $\mathrm{sp}^{2}$ carbon codoped with $\mathrm{B}$ and $\mathrm{N}$ atoms is entirely different when $\mathrm{B}$ and $\mathrm{N}$ are bonded together or located separately, which leads to distinct activities for, e.g., the oxygen reduction reaction ${ }^{8,9}$ Central to the exploration of 2-D materials, their hybrids and the degree to which their emergent properties can be tailored at the atomic level is therefore the question of what type of dopant (and codopant) bond arrangements form in graphene and what are the electronic effects of specific bond motifs.

To this end we combine synchrotron-based x-ray techniques, including X-ray Photoelectron Spectroscopy (XPS), Near Edge X-ray Absorption Fine Structure (NEXAFS), and photoemission threshold measurements, with scanning tunneling microscopy (STM) and theoretical calculations within the framework of Density Functional Theory (DFT) to characterize boron and nitrogen $(\mathrm{B} / \mathrm{N})$ co-doped graphene grown by chemical vapor deposition (CVD) methods. The suite of techniques reveals an atomic-level picture of individual B-N dopant structures and their effects on the electronic structure of single layer graphene. We find that B and $\mathrm{N}$ dopant atoms cluster into specific BNC dopant structures rather than being randomly distributed with well-separated, individual $\mathrm{B}$ and $\mathrm{N}$ graphitic dopants. First principles calculations show there is a thermodynamic driving force for $\mathrm{B}$ and $\mathrm{N}$ atoms to form local $\mathrm{BNC}$ dopant clusters; in experiments performed here, kinetics apparently limit the segregation of large boron nitride domains within the graphene sheet, an effect that has been experimentally observed elsewhere ${ }^{2}$. We find that the macroscopic doping effect (p- or n-type) is largely ruled by the $\mathrm{B}: \mathrm{N}$ ratio in the films ( $\mathrm{B}>\mathrm{N}$, p-type; $\mathrm{B}<\mathrm{N}$, n-type; $\mathrm{B}=\mathrm{N}$, neutral), which can be understood based on the comparable electron withdrawing and donating effects of substitutional (graphitic) $\mathrm{B}^{23-25}$ and $\mathrm{N}$ dopants, ${ }^{13,21,22}$ respectively. Accordingly, for boron and nitrogen co-doped graphene with a 1:1 B: 
$\mathrm{N}$ ratio, we observe no shift in work function relative to pristine (undoped) graphene in photoemission threshold measurements. First principles calculations indicate that the specific B$\mathrm{N}$ dopant configurations visualized and characterized in this work, and $\mathrm{B} / \mathrm{N}$ co-dopant structures in general, open a small gap in the electronic band structure of graphene. These findings contribute fundamental insight into the fabrication and electronic structure effects of $\mathrm{B} / \mathrm{N}$ codoped graphene and the formation of atom-scale boron-nitrogen-carbon (BNC) clusters within a single graphene sheet. These newfound understandings have implications for the atomic-level design of carbon-based nanomaterials with the potential to impact post-silicon electronics and offer non-platinum based (electro)catalysts and catalyst supports with enhanced efficiency for clean energy applications.

\section{RESULTS AND DISCUSSION}

\section{Chemical Vapor Deposition (CVD) growth}

Boron and nitrogen co-doped graphene $(\mathrm{B} / \mathrm{NG})$ films were grown on polycrystalline copper foils using a mixture of $\mathrm{CH}_{4}, \mathrm{H}_{2}$, and $\mathrm{B}_{2} \mathrm{H}_{6}\left(1 \%\right.$, balance $\left.\mathrm{H}_{2}\right)$ and $\mathrm{NH}_{3}$ gases at $1000{ }^{\circ} \mathrm{C}$ in a quartz tube furnace. $\mathrm{B}_{2} \mathrm{H}_{6}$ and $\mathrm{CH}_{4}$ were flowed together at rates of $1 \mathrm{sccm}$ and $1.8 \mathrm{sccm}$, respectively, through the chamber for 6 seconds (s) and then both were stopped. Afterwards the chamber was continually flushed with the $\mathrm{H}_{2}$ at $100 \mathrm{sccm}$ for $60 \mathrm{~s}$, a combination of $\mathrm{NH}_{3}$, at $3 \mathrm{sccm}$, and $\mathrm{CH}_{4}$, at $1.8 \mathrm{sccm}$, gases was then introduced for $30 \mathrm{~s}$. After $30 \mathrm{~s}$ the $\mathrm{NH}_{3}$ was stopped and the $\mathrm{CH}_{4}$ continued for the remainder of the time. For comparison, graphene was also prepared using only $\mathrm{B}_{2} \mathrm{H}_{6}(1 \mathrm{sccm})$ as the dopant gas (no $\mathrm{NH}_{3}$ flow); these samples are denoted BG1 and BG2 in the text where BG1 differs from BG2 in that during growth the reaction environment was diluted 
with Ar. Pristine graphene (PG) sheets were also grown using $\mathrm{CH}_{4}$ and $\mathrm{H}_{2}$ under the above conditions for comparison.

Following growth, each $\mathrm{B} / \mathrm{NG}$ sample was cut in half. One half was transferred in Argon atmosphere to an ultrahigh-vacuum (UHV) end station for x-ray spectroscopy measurements. The other half was transferred from ambient conditions to a UHV chamber for STM measurements and annealed at $350{ }^{\circ} \mathrm{C}$ for $5 \mathrm{~h}$ to clean the sample surface. BG and PG samples were transferred in $\mathrm{N}_{2}$ atmosphere to the UHV end station for x-ray measurements.

\section{Core level spectroscopy}

We previously demonstrated that core-level resonant x-ray spectroscopy is a sensitive probe of chemical bonding and electronic structure of chemical dopants introduced in single-sheet graphene films..$^{22}$ X-ray Photoelectron Spectroscopy (XPS) is the seminal analytical tool for atom specific information on local coordination environments and atomic concentration and has been extensively applied to study chemical doping of graphene $e^{8,10-12,22,26}$ as well as bonding in boron nitride carbide (BNC) hybrid films. ${ }^{38-40}$ In Figure 1 we show XPS measured at the carbon (C 1s), boron (B 1s) and nitrogen (N 1s) K-edges used to quantify the atomic concentration of boron and nitrogen dopants and identify dopant bond environments for $\left(\mathrm{B}_{2} \mathrm{H}_{6}+\mathrm{NH}_{3}\right)$-grown $(\mathrm{B} / \mathrm{NG})$ and $\mathrm{B}_{2} \mathrm{H}_{6}$-grown graphene (BG1 and BG2). Atomic concentrations are obtained from the core level photoemission intensity for each element normalized by the atomic sensitivity factor (photoionization cross-section) at a given photon energy.

In our co-doped graphene sheets, we find a $\mathrm{B}$ : $\mathrm{N}$ atomic ratio of unity with $\mathrm{B}$ and $\mathrm{N}$ concentrations ranging from $\sim 1-5 \%$, as shown in Figure 1 inset and in Table 1 (details provided in the Methods section). No boron or nitrogen signal is observed for pristine graphene (see Fig. 
1d). For all samples, C 1s XPS data (Fig. 1, center) shows the characteristic photoemission line of pristine graphene, an asymmetric peak centered at $284.5 \mathrm{eV}$ arising from $\mathrm{C}=\mathrm{C}$ bonds The concentration of B and $\mathrm{N}$ dopants in our co-doped samples is not high enough to introduce new features in the C 1s XPS relative to pristine graphene (PG); aside from a small shoulder at $286.2 \mathrm{eV}$ for the $\mathrm{B}_{2} \mathrm{H}_{6} / \mathrm{NH}_{3}$-grown samples $(\mathrm{B} / \mathrm{NG})$. We attribute this shoulder to $\mathrm{C}=\mathrm{N}$ bonds $^{41,42}$, which may be due to a dilute population (sub-percent level) of graphitic N-dopants. ${ }^{22}$

What structural motifs do the comparable concentrations of boron and nitrogen atoms form in graphene? Do the B and N dopants distribute randomly as individual substitutional (graphitic) dopants or prefer to arrange into B-N clusters? We glean our first insight to this question from B 1s (Fig. 1 left) and N 1s (Fig. 1 right) XPS measurements of co-doped graphene (B/NG). Fig. 1 shows broad (FWHM 1.4-1.6eV), fairly symmetric B 1s and N 1s XPS peaks, centered at 189.6 $\mathrm{eV}$ for $\mathrm{B} 1 \mathrm{~s}$ and $\sim 398.8 \mathrm{eV}$, respectively, characteristic of BNC bond structures ${ }^{43-45}$ Both the boron (Fig. 1 left) and nitrogen XPS (Fig. 1 right) are best fit by a main peak (189.52-189.65 eV for B 1s and 386.6-398.86 eV for $\mathrm{N} 1 \mathrm{~s})$ and a weak, higher binding energy component (190.0190.2 eV (B 1s) and 399.3-399.7 (N 1s)), both of which we assign to BNC bond structures as established in the literature. ${ }^{43-46}$ The low intensity, broad peaks at higher binding energy in the B 1s data (Fig. 1 left) may also include a small contribution from substitutional $\mathrm{sp}^{2}$-hybridized $\mathrm{B}=\mathrm{N}$ bonds, although this peak is most typically observed closer to $191 \mathrm{eV}$. $^{2,48,49}$

Importantly, the (broader) width and binding energy of the B $1 \mathrm{~s}$ and $\mathrm{N}$ 1s photoemission lines for the co-doped films are not only characteristic of BNC hybrid bond configurations, but also distinctly different from that of h-BN,,$^{2,47,48}$ boron carbide, ${ }^{49,50} \mathrm{~B}$-doped graphene, ${ }^{23,24}$ and sp ${ }^{2} \mathrm{~N}=\mathrm{C}$ bonds in carbon nitride or $\mathrm{N}$-doped graphene; ${ }^{41,42}$ the reported range for each bonding motif is indicated with labeled brackets in Fig. $1 \mathrm{~b}$ and c. Both Jacobsohn et al ${ }^{49}$ and Jiménez et al. ${ }^{50}$ 
report B 1s binding energies for B-C bonds in boron carbide below $189 \mathrm{eV}(188.7,188.4,188$ $\mathrm{eV}$, and as low as $187 \mathrm{eV}$ ), well below main line of our B 1s peak observed at $\sim 189.6 \mathrm{eV}$ (Fig. 1 left). Taking $188.7 \mathrm{eV}$ as an upper limit for a B-C binding energy and fitting the B 1s XPS yields a very low intensity peak, indeed (not shown). Together with the 1:1 B: $\mathrm{N}$ composition ratio, the N 1s and B 1s XPS peak positions (Fig. 1), clearly indicates that isolated boron and nitrogen dopants cannot be prevalent in the co-doped graphene films.

Incident polarization angle dependent NEXAFS measured at the boron (B 1s), carbon (C 1s), and nitrogen (N 1s) K-edges in Figure 2 provides a direct, element-specific probe of bond type (e.g. B-N, C-N, B-C) and orientation in $\mathrm{B} / \mathrm{N}$ co-doped graphene, as well the effect of $\mathrm{B} / \mathrm{N}$ codoping on the electronic structure of graphene. C 1s NEXAFS (Fig. 2 center) shows that the 2-D nature and periodicity in the electronic structure of graphene is essentially unperturbed by this level of $\mathrm{B} / \mathrm{N}$ co-doping, which is too low to introduce discernible features in the spectra due to C-N and/or C-B bond formation, as observed in the XPS data (Fig. 1). In particular, the data for pristine and doped graphene samples are nearly identical, and show the spectral fingerprints of $\mathrm{sp}^{2}$-hybridized carbon sheets. ${ }^{51,52}$ These fingerprints include peaks at 285.4 and $292 \mathrm{eV}$, corresponding to $1 \mathrm{~s}->\pi^{*}$ and $1 \mathrm{~s}->\sigma^{*} \mathrm{C}=\mathrm{C}$ bond resonances of graphitic carbon, respectively. ${ }^{51,52}$ The strong and opposite angular dependence of the intensity of these peaks characteristic of planar sheets is observed for all samples, indicating that the graphene lattice does not buckle with $\mathrm{B} / \mathrm{N}$ doping under these conditions. In addition, the sharp core-hole excitonic $\sigma^{*}$ feature at 291.5 $\mathrm{eV}$, a fingerprint of long-range periodicity in the electronic structure of graphene, is preserved with doping; this feature is also observed in graphite. ${ }^{51,52}$ The B 1 s and $\mathrm{N}$ 1s NEXAFS for the co-doped samples display a strong angular dependence following that of the C $1 \mathrm{~s}$ data; the $\pi^{*}$ intensity is greatest at an incident angle of $20^{\circ}$, when the electric field (E-) vector of the 
incoming x-ray beam is nearly perpendicular to the surface and minimum at $90^{\circ}$, when the Evector is oriented in the surface plane; the $\sigma^{*}$ peaks show the opposite dependence. This indicates that the $\pi^{*}$ orbitals are oriented along the surface normal and the nitrogen and boron dopants form planar bonds in the graphene lattice (see Fig. 2 schematic inset), as predicted by theory. ${ }^{53}$

Comparison of the B 1s and N 1s NEXAFS resonances for B/NG (Fig. 2c) with that of h-BN (Fig. 2d), boron carbide and BCN films provide further insight on the structural details of the dopant bond motifs. The strong resemblance between the $\mathrm{B} 1 \mathrm{~s}$ and $\mathrm{N} 1 \mathrm{~s}$ data for $\mathrm{B} / \mathrm{NG}$ and h$\mathrm{BN}^{54-55}$ (Fig. 2c,d) reveals that a significant concentration of $\mathrm{sp}^{2}$-hybridized B-N bonds indeed form in our co-doped graphene samples, while specific differences indicate that these bonds are incorporated into BNC structures, as observed with XPS (Fig. 1). In particular, B/NG displays B 1s NEXAFS resonances characteristic of h-BN, including a sharp B $1 \mathrm{~s}->\pi^{*}$ peak at $\sim 192 \mathrm{eV}$ and several B 1s-> $\sigma^{*}$ resonances, including a broad feature split two components (of similar intensity in h-BN), $\sigma_{1}{ }^{*}$ at $198 \mathrm{eV}$ and $\sigma_{2}{ }^{*}$ at $199.5 \mathrm{eV}^{55}$ (Fig. 2c,d). However, as observed in boron and nitrogen co-doped nanotubes with $\mathrm{CB}_{\mathrm{x}} \mathrm{N}_{\mathrm{y}}$ bonding ${ }^{9}$ and $\mathrm{BNC}$ hybrid films, $\mathrm{B} / \mathrm{NG}$ shows a higher $\sigma_{2}{ }^{*}: \sigma_{1}{ }^{*}$ intensity ratio relative to h-BN, and the intensity of the h-BN $\sigma_{3}{ }^{*}$ resonance at $\sim 204 \mathrm{eV}$ is strongly damped, if even detectable, in B/NG (Fig. 2 left). The B 1s NEXAFS for B/NG is, in fact, rather typical of BNC hybrid films ${ }^{38-40,43-45}$ and distinctly different from that of boron carbide, which is characterized by a $1 \mathrm{~s}->\pi^{*}$ transition at lower photon energy $(\sim 190.9 \mathrm{eV}) .{ }^{50}$ Accordingly, we attribute the slight downward shift of the B $1 \mathrm{~s} \pi^{*}$ resonance of $\mathrm{B} / \mathrm{NG}$ compared to h-BN (191.6 vs. $192 \mathrm{eV}^{2,48}$ ), to the presence of $\mathrm{B}-\mathrm{C}-\mathrm{N}$ bonds and the lower electronegativity of $\mathrm{C}$ relative to $\mathrm{N}$ (Fig. 2c, d (left)). ${ }^{51}$ 
The corresponding $\mathrm{N}$ 1s NEXAFS (Fig. 2, right) further supports clustering of $\mathrm{N}$ and $\mathrm{B}$ dopants into local BNC bond structures in boron and nitrogen co-doped graphene. In the case of randomly distributed, well separated individual $\mathrm{N}$ and $\mathrm{B}$ dopants, the $\mathrm{N}$ 1s NEXAFS would closely resemble the spectrum for substitutionally $\mathrm{N}$-doped graphene, with a sharp $\pi^{*}$ and $\sigma^{*}$ peak at $\sim 400.8 \mathrm{eV}$ and $405 \mathrm{eV}$, respectively. ${ }^{22}$ Instead, as for the boron core-level data (Fig. 2 left), the $\mathrm{N}$ 1s NEXAFS for $\mathrm{B} / \mathrm{NG}$ is very similar to that of $\mathrm{h}-\mathrm{BN}$, with the main differences pointing to the formation of both N-C and N-B bonds. Specifically, B/NG displays the main N 1s spectral fingerprints of h-BN, including a $\pi^{*}$ resonance at $401.4 \mathrm{eV}$ with a shoulder at higher photon energy and two main $\sigma^{*}$ features, centered at $\sim 408.8\left(\sigma_{1}^{*}\right)$ and $\sim 416 \mathrm{eV}\left(\sigma_{2}^{*}\right)$, each with significant fine structure. ${ }^{2,48}$ The increased intensity between/broadening of $\sigma_{2}^{*}$ and $\sigma_{2}^{*}$ for $\mathrm{B} / \mathrm{NG}$ relative to $\mathrm{h}-\mathrm{BN}$, is in particular, a fingerprint of $\mathrm{B}-\mathrm{C}-\mathrm{N}$ bonding. ${ }^{43-45}$ The $\mathrm{N} 1 \mathrm{~s} \pi^{*}$ resonance of $\mathrm{B} / \mathrm{N}$ codoped graphene (Fig. 2a-d, right) lacks the shoulder at lower $(\sim 0.5 \mathrm{eV})$ photon energy observed for h-BN (Fig. 2d, right). The difference in energy position of the $\sigma^{*}$ resonances (relative to the ionization potential (IP)) between the codoped and h-BN sample reflect differences in bond length ("bond length with a ruler"). ${ }^{51}$ A shorter B-N bond will move the $\sigma^{*}$ resonance further from the IP and vice versa. Since the intensity of the photoemission (Fig. 1) and NEXAFS resonances (Fig. 2) scale with the concentration of the specific bonds from which they arise, the x-ray data (Figs. 1-2) not only confirms formation of substitutional B-N-C dopant structures as the dominant dopant bond motif, even at low doping levels, but also excludes the possibility of formation of distinct boron nitride domains ${ }^{2}$ or a detectable population of isolated substitutional B or $\mathrm{N}$ dopants in our co-doped graphene samples.

The clustering of B- and N-dopants in graphene is not surprising given that the favorable segregation of B-N and C-C bonds into distinct domains ${ }^{2}$ has represented a long-standing 
challenge to synthesis of BNC hybrid films. In fact, we have demonstrated and characterized a large series of $\mathrm{B}_{2} \mathrm{H}_{6}$-grown graphene films where the dominant ( $\left.>80 \%\right)$ doping form is individual substitutional B-dopants, ${ }^{25}$ our studies revealed that, in general, the quality of the B-doped films was far more sensitive to the dopant gas $\left(\mathrm{B}_{2} \mathrm{H}_{6}\right)$ concentration and flow rate, when compared to $\mathrm{N}$-doped graphene films. In some cases, the favorability of B-N bond formation in carbon films is strong enough to facilitate incorporation of $\mathrm{N}$ into the graphene lattice for $\mathrm{B}_{2} \mathrm{H}_{6}$-grown samples without flow of $\mathrm{NH}_{3}$ during growth, due to low levels of nitrogen contamination that persist in the CVD growth tubes even after scrubbing (BG1 and BG2 in Fig 1). In N-doped $\left(\mathrm{NH}_{3}\right.$-grown) graphene, the $\mathrm{N}$ 1s NEXAFS signal from the N-dopants overwhelms the low intensity contribution from $\mathrm{N}$ contaminants, however $\mathrm{N}$ 1s NEXAFS and XPS of pristine graphene reveal a small signal from parasitic nitrogen. ${ }^{22}$ In fact, some $\mathrm{B}_{2} \mathrm{H}_{6}$-grown graphene sheets show a significant nitrogen signal, with both the $\mathrm{B}$ 1s and $\mathrm{N}$ 1s data for these films (BG1 and BG2 in Fig. 2a,b) strikingly similar to that of the $\mathrm{B}_{2} \mathrm{H}_{6}+\mathrm{NH}_{3}$ doped (B/NG) samples (Fig. 2c). Collectively, the data for the series shows that as the concentration of nitrogen increases with that of boron, the double peak feature in the shape resonance in the B $1 \mathrm{~s}$ NEXAFS $\left(\sigma_{1}{ }^{*}\right.$ and $\sigma_{2}{ }^{*}$ in Fig. 1c, d (left)), which contains a second order contribution from nitrogen K-edge absorption, increases in intensity due to the formation of B-N bonds.

It is also clear from the $\mathrm{x}$-ray data (Figs. 1-2) that flowing $\mathrm{NH}_{3}$ after $\mathrm{B}_{2} \mathrm{H}_{6}$ co-dopes the graphene sheet with both boron and nitrogen and that although the B: $\mathrm{N}$ ratio is always close to unity, the total dopant concentration varies with growth conditions. In a perfectly random distribution of 1\% B and 1\% $\mathrm{N}$ dopants in graphene (e.g. BG1 in Fig. 1 and Fig. 2), we would expect a $\sim 3 \%$ probability of nitrogen and boron atoms occupying neighboring sites to form a local B-N bond. 
(Consider 100 sites, the first one of which is occupied by B; then there are three nearest neighbor sites available resulting in a local B-N bond while the remaining 96 do not result in a B-N bond.) Since the intensity of the specific peaks in the x-ray scales linearly with the concentration of the bond type from which it arises (the measured signal represents this weighted sum), a 3\% probability of B-N bond formation is far too low to account for the excess BNC contribution to the signal measured in Figs. 1 and 2. There must be a thermodynamic and/or kinetic driving force for the clustering of B and $\mathrm{N}$ into local BNC dopant bond motifs; we will return to this in the following.

\section{Scanning Tunneling Microscopy}

To directly visualize in real space the doping forms of Boron and Nitrogen atoms in the B-N co-doped graphene monolayer films, we performed low temperature scanning tunneling microscopy (LT-STM) measurements on the as grown co-doped graphene films on copper foil. In general, the doping process increases the roughness of the substrate making STM measurements challenging (see supporting info for an example of a rough substrate). Due to this, STM topographies with atomic-level resolution were not obtained for the $\mathrm{B}_{2} \mathrm{H}_{6} / \mathrm{NH}_{3}$-grown samples (B/NG). However, we were able to resolve the atomic-level topography for $\mathrm{B}_{2} \mathrm{H}_{6}$-grown samples, which exhibit very similar x-ray spectroscopic signatures (BG1 and BG2 in Figs. 1 and 2). The measurements were taken in Omicron ultra-high vacuum (UHV) LT-STM with a base pressure of $\sim 5.0 \times 10^{-12}$ torr at $77 \mathrm{~K}$. After the synthesis of the films, they were transferred from the CVD furnace through ambient atmosphere to the UHV STM chamber. Before STM measurements, a gentle annealing procedure at $\sim 350 \mathrm{C}$ for $\sim 4$ hours in UHV condition was applied, in order to eliminate any physisorbed species on the surfaces of the graphene films. 
A large number of surveys over the sample surfaces prove the presence of dopants in the graphene lattice, which appear as bright features in STM topography and are not present in the pristine graphene film. Closer investigations indicate multiple structures of these bright features, associated with different dopant types and doping forms. A good example of such detailed investigations is shown in Fig. 3(a), which shows an STM topography of $10 \times 10 \mathrm{~nm}^{2}$ taken on asgrown B-N co-doped graphene films on copper foil. The bright features in this figure can be categorized into three groups. The image for the first kind, highlighted by the red arrow in Fig. 3A (lower right), shows a hollow-centered triangle made by three bright spots at the apexes. The image for the second type, highlighted by the white arrow, displays a solid-centered triangle. Our previous studies on Nitrogen-doped and Boron-doped graphene films have demonstrated that the first type of feature (red arrow) results from single graphitic $\mathrm{N}$ dopants ${ }^{21}$ and the second kind (white arrow) is produced by single graphitic B dopants. ${ }^{25}$

In addition to these two triangular features previously discussed, ${ }^{21,25}$ other, more complicated types are also observed in two main forms; these features, which we denote STM1 and STM2 are highlighted in boxes in Fig. 3a labeled "b" and "c" respectively. These types of complex features are observed at roughly a $0.1 \%$ level in the sample, as seen from large area topographs (see supporting information for details). High-resolution STM topographies show more details of STM1, displayed in Fig. 3(b) and STM2 in Fig. 3(c). We can overlay the honeycomb lattice across the STM1 and STM2, images, as shown in Fig. 3(b) and 3(c), registering from the unperturbed graphene lattice a couple of nanometers away from the features. In previous experiments, single graphitic $\mathrm{N}$ dopants were seen to enhance the electronic density of states most at its three first nearest neighbors (resembling a hollow-centered triangle) ${ }^{21}$ while single graphitic B dopants showed the strongest enhancement at the boron site itself (resembling a 
solid-centered triangle). ${ }^{25}$ Motivated by this, and the nature of the variation in intensity of this third type of feature, we propose models with a combination of both $\mathrm{N}$ and $\mathrm{B}$ dopants to explain the features as shown in Fig. 3(b) and 3(c), as indicated by green dots for $\mathrm{N}$ dopants and yellow dots for B dopants. To confirm the validity of the proposed models, we simulated the STM topographies of the B-N co-doped models in Fig. 3(b) and (c) based on DFT calculations, and show the results in Fig. 3(d) and (e). As we can see, the simulated results reproduce the experiment data very well, confirming that the complicated features observed (STM1 and STM2) do indeed result from the co-existence of $\mathrm{B}$ and $\mathrm{N}$ dopants within atomic distances. Comparison of computed STM topographies for a series of possible B-N co-dopant structural motifs (Supplementary Figures S1-S2) with the experimental data further supports the assignment of STM1 and STM2 features to the specific B/N structures shown in identified in Fig. 3. Further evidence of the atomic arrangements in these doping complexes can potentially be provided by scanning tunneling spectroscopy (STS) imaging measurements. Indeed, such measurements [ref 28] have recently been performed on $\mathrm{N}$-doped graphene which demonstrate the local changes to the DOS caused by dopants. In the samples studied so far, spectroscopy measurements were not reliably obtained due to the presence of contamination that made STS image acquisition too noisy. Improved sample cleaning procedures are necessary to enable such measurements in the future.

The individual B-N dopant structures visualized with STM (Fig. 3) fit well with the $1: 1 \mathrm{~B}: \mathrm{N}$ ratio determined by XPS (Fig. 2, Table 2) and the substitutional BNC bonds identified with NEXAFS (Fig. 1). The STM structures further confirm that the shift in B 1s and N 1s XPS peaks to lower and higher binding energy, respectively, relative to h-BN (Fig. 2b,c) may be attributed to substitutional (graphitic) co-dopants in the graphene lattice, due to the lower/higher 
electronegativity of B/N relative to C. The presence of B-B bonds in STM2 (Fig. 3c) may also contribute to this effect in the B 1s XPS data (Fig. 2b). ${ }^{56}$

\section{Electronic effects of specific BNC dopant bond configurations}

Having visualized and characterized the atomic-level $\mathrm{B} / \mathrm{N}$ dopant bond configurations in single layer graphene, the remaining question is how do the different dopant structural motifs affect the electronic structure of co-doped graphene? With the excellent agreement between experimental and simulated STM images establishing the basic atomistic details of these dopant bonding motifs, we use DFT calculations for extended graphene sheets with supercell dimension $(7 \times 7)$ that corresponds to $\sim 1 \%$ for a single $\mathrm{N}$ or B dopant (details in the Method section and Supporting Information). We compute the formation energy of different $\mathrm{B} / \mathrm{N}$ dopant motifs, including those identified here (STM1 and STM2 in Fig. 3(b) and (c)) and several others visualized in Fig. S1. We also investigate the corresponding shift in work function as well as the fractional charge added (withdrawn) per net number of dopant atoms for non-neutral combinations, as shown in Table 1.

The formation energy for the different $(\mathrm{B}, \mathrm{N}$ and $\mathrm{B} / \mathrm{N})$ dopant structures in Table 1, computed relative to a single (B or $\mathrm{N}$ ) graphitic dopant and pristine graphene, clearly show there is an energetic driving force for the formation of local $\mathrm{BN}$ structures in boron and nitrogen co-doped graphene with $\mathrm{B}$ and $\mathrm{N}$ in nearest neighbor configurations. Specifically, there is a large stabilization of the B-N nearest neighbor pairs (by $2.35 \mathrm{eV}$ ) while $\mathrm{B}-\mathrm{B}$ and $\mathrm{N}-\mathrm{N}$ pairs are unstable. This is fully consistent with results in the literature that point to substantially stronger C-C and B-N bonds over B-C, B-B, N-C and N-N bonds. ${ }^{53,57,58}$ Furthermore, analysis of structures with two or three separated $\mathrm{B} / \mathrm{N}$ pairs shows a formation energy approximately linear 
in the number of such pairs. The other structural motifs, such as STM2, have B-N nearest neighbor pairs, but with different organization and shared sites (e.g., the shared B site for STM2 in Fig. 3c). Some of them also have excess associated B. These all show a similar strong formation energy that is primarily attributable to the attraction between $\mathrm{B}$ and $\mathrm{N}$, balanced by repulsion between near neighbor B and $\mathrm{N}$ pairs. Yuge supplied a more detailed analysis of the thermodynamics of phase formation using a cluster expansion approach. ${ }^{57}$ Our results are generally consistent with his observation that at temperatures typical of the present experimental growth conditions $\left(100{ }^{\circ} \mathrm{C}\right)$, thermodynamics favors phase segregation between hexagonal $\mathrm{BN}$ and graphene.

In view of these results, our observation of extended $\mathrm{B} / \mathrm{N}$ structural motifs at a rate that substantially exceeds random statistical occurrence can easily be understood. To illustrate this, refer to the example described above showing a $3 \%$ probability for $\mathrm{B}-\mathrm{N}$ bond formation in a sample with 100 sites and random placement of one each of B and N. If the large, computed formation energy for a $\mathrm{B} / \mathrm{N}$ dopant pair $(-2.35 \mathrm{eV}$, Table 1), when incorporated into the Boltzmann factor for the B-N dopant pair in the partition function for the same sample at $\mathrm{T}=1000 \mathrm{C}$, shows that in a sample of a 100 lattice sites with one $\mathrm{B}$ and one $\mathrm{N}$, the probability that individual boron and nitrogen dopant atoms will occupy neighboring sites in graphene is essentially $100 \%$. Isolated B and $\mathrm{N}$ centers would not be observed. With more B and/or N atoms available locally, the equilibrium solution is formation of more extended local BN complexes. Naturally, the countervailing factor is the extent to which individual atoms are locked into place during the actual growth process, with insufficient subsequent mobility to sample lower energy configurations. Considering that the STM2 configuration, readily observed, is not the lowest energy motif available for three B-N pairs, this suggests that kinetic limitations and the details of 
the actual growth mechanism play a significant role. Furthermore, the STM data (Fig. 3) clearly shows examples of isolated $\mathrm{B}$ and $\mathrm{N}$ centers in coexistence with the BN complexes.

Table 1 also shows that the doping effect (p- or n-type) is determined by the $\mathrm{B}$ : $\mathrm{N}$ ratio in codoped graphene $(\mathrm{B}>\mathrm{N}$, p-type; $\mathrm{B}<\mathrm{N}$, n-type; $\mathrm{B}=\mathrm{N}$, neutral $)$, which we understand based on the comparable electron withdrawing and donating effects of substitutional (graphitic) B and $\mathrm{N}$ dopants, respectively. ${ }^{21,25}$ This is found directly from the band structure and the position of the Fermi energy relative to the Dirac point. To make a more direct connection with experiment, this is also demonstrated by calculated changes in the work function. As seen in other calculations, ${ }^{28,29,35,36}$ the presence of the dopant atoms opens gaps at different parts of the band structure, relative to pristine graphene, in particular at the Dirac point. This is illustrated for STM1 and STM2 studied here in Fig. 4. For the case of a balanced number of B and N dopants, the gap at the Dirac point straddles the Fermi energy (taken to be at the mid-point of the gap), as in Fig. 4a. For an unbalanced case, the Fermi energy is displaced by the doping effect (Fig. 4b). Furthermore, these gaps increase with the relative concentration of foreign atoms. The gap in Fig. 4a $(0.29 \mathrm{eV})$, for a motif with $3 \mathrm{~B} / \mathrm{N}$ pairs in the 98 atom super cell is about three times that found for a single $\mathrm{B} / \mathrm{N}$ pair in the same cell $(0.10 \mathrm{eV})$. Other dopant-induced changes may also be significant. For example, in Fig. 4b, gaps also open near the M point and alter the dispersion of the bands. This can be seen clearly for the band between $\mathrm{M}$ and $\mathrm{K}$ immediately above the Fermi energy. This is the band that hosts the holes induced by the net p-type doping in this case. The Fermi energy is close to a saddle point in the band. Correspondingly the Fermi energy shift will deviate from that expected for simple linear dispersion.

Clear trends still emerge in the doping range studied here (up to a net of $2 \%$ ). This is illustrated in Figure 5, which shows the computed change in work function versus the net doping 
(concentration of $\mathrm{B}$ minus concentration of $\mathrm{N}$ ). The work function change reflects both the filling of the bands (the Dirac point shift or a quantum capacitance) and an electrostatic effect. The latter is smaller and has opposite sign. The sign of the electrostatic contribution can be understood as follows. For $\mathrm{N}$ dopants, the p-orbital has smaller radius, pulling negative charge more tightly in towards the plane of the graphene layer, a repulsive effect that reduces the work function. For B dopants, the effect is opposite: the p-orbital has a larger radius, pushing positive charge outwards from the plane of the graphene layer and increasing the work function. In addition, small deviations from planarity driven by local strain effects in the $\mathrm{B} / \mathrm{N}$ complexes also contribute. For a more quantitative view Fig. S2 shows that the overall change in the work function is about $75 \%$ of the band filling effect (shift of the Dirac point).

We can also connect these results to our earlier measurements and calculations for nitrogen dopants that incorporate into graphene with several structural motifs including graphitic, as considered here up to now (N3) as well as in motifs involving complexes with vacancies in which the $\mathrm{N}$ center is two-fold bonded to the carbon network (pyridinic, N2) and one fold bonded (N1). Each motif has a specific spectral signature and in contrast to N3, which is n-type, the N1 and N2 were found to be p-type. Details are provided in Supplementary Information (see Figs. S3-S4, Table S1, and ref. 22). The measured and computed work function change as a function of net implied doping is added to Fig. 5.

\section{CONCLUSIONS}

To summarize, we have fabricated boron and nitrogen co-doped single layer graphene grown on copper foil, characterized and visualized substitutional $\mathrm{B} / \mathrm{N}$ co-dopant structures at the atomic 
level, and correlated the specific dopant bond motifs with electronic structure effects. We find that boron and nitrogen cluster into local BNC dopant bonding configurations rather than being randomly distributed as individual graphitic dopants in atomic sheet of carbon atoms. First principles calculations show that BNC dopant bond structures in general, as well as the specific atomic arrangements identified here, open a small band gap in graphene. Moreover, calculations and measurements connecting work function change with dopant type and concentration show the that electronic effect of the dopant structural motif is determined by the p-:n-type dopant ratio $(\mathrm{B}>\mathrm{N}$ is p-type, $\mathrm{B}<\mathrm{N}$ is $\mathrm{n}$-type and $\mathrm{B}=\mathrm{N}$ is neutral in co-doped graphene; the carrier concentration is $\mathrm{N}$-doped graphene similar shifts with the relative concentration of $\mathrm{p}$ - and n-type $\mathrm{N}$ doping forms). This result is supported by a zero shift in work function obtained with photoemission threshold measurements for boron and nitrogen co-doped samples with a B: $\mathrm{N}$ ratio of unity determined from XPS measurements. Electrostatic effects based on the size of the dopant atom relative to the carbon host also play a role. We expect that the understanding obtained in this work will help pave the way to next generation, carbon-based electronics and catalysts with an electronic band structure and carrier concentration optimized at the atomic level for a desired application.

\section{METHODS}

Core level x-ray spectroscopy. NEXAFS and XPS measurements were performed at the wiggler source beamline 10-1 at the Stanford Synchrotron Radiation Lightsource (SSRL), and at the NIST (bending magnet source) beamline U7A of the National Synchrotron Light Source (NSLS) in ultra-high vacuum (better than $10^{-9}$ torr) endstations designed for surface and solid 
state-experiments. The reference absorption intensity (I0) of the incoming x-ray beam, measured on a gold-coated mesh positioned just after the refocusing optics, was measured simultaneously and used to normalize the spectra to avoid any artifacts due to beam instability. Polarizationdependent $\mathrm{C}$ 1s, $\mathrm{B}$ 1s and $\mathrm{N}$ 1s NEXAFS data were obtained by changing the angle between the incoming $\mathrm{x}$ - ray beam from near-parallel $20^{\circ}$ and normal $\left(90^{\circ}\right)$ incidence. A linear background was subtracted from the NEXAFS data as determined from a region before the absorption edge and the spectra were normalized by area in the post-continuum region where no absorption transitions appear (between 320 and $330 \mathrm{eV}$ for carbon; 420 and $430 \mathrm{eV}$ for nitrogen and 220230 for boron). K-edge NEXAFS collection was performed in the partial electron yield (PEY) mode at U7A, with a grid biases of $-200 \mathrm{~V},-280 \mathrm{~V}$ and $-150 \mathrm{~V}$ selected for $\mathrm{C} 1 \mathrm{~s}, \mathrm{~N}$ 1s, and B $1 \mathrm{~s}$ measurements, respectively, in order to the surface sensitivity of the measurement. At SSRL beamline 10-1, NEXAFS was measured in total electron yield (TEY) mode, which measures the total drain current from the sample. XPS measurements were obtained at multiple photon energies to improve the statistics on the quantification of B: C: $\mathrm{N}$ atomic concentration ratios.

SSRL beamline 10-1 has a spherical grating monochromator and the focused beam has a spot size of $<1 \mathrm{~mm}^{2}$. NSLS U7A utilizes a toroidal spherical grating monochromator and the focused beam has a spot size of $\sim 1.5 \mathrm{~mm}^{2}$. XPS was measured SSRL beamline $10-1$ at an incident angle of $20^{\circ}$, while an incident angle of $54^{\circ}$ was found to be least sensitive to any surface inhomogeneity caused by flexibility of the $\mathrm{Cu}$ foil in photoemission threshold measurements. Measurements were repeated at multiple spots across the sample surface (at least 2 for NEXAFS, 4 for XPS and 20 for photoemission threshold measurements). 
The B 1s, C 1s and N 1s binding energies for the individual samples were calibrated by the C 1s photoelectron peak for pristine graphene $(284.5 \mathrm{eV})$, at the excitation photon energy used for measurement $(280,410,650 \mathrm{eV})$. The atomic concentration of $\mathrm{B}, \mathrm{C}$ and $\mathrm{N}$ in each graphene sample was estimated from $\left(\mathrm{I}_{\mathrm{B} 1 \mathrm{~s}} / \sigma_{\mathrm{B} 1 \mathrm{~s}}\right) /\left[\left(\mathrm{I}_{\mathrm{B} 1 \mathrm{~s}} / \sigma_{\mathrm{B} 1 \mathrm{~s}}\right)+\left(\mathrm{I}_{\mathrm{N} 1 \mathrm{~s}} / \sigma_{\mathrm{N} 1 \mathrm{~s}}\right)+\left(\mathrm{I}_{\mathrm{C} 1 \mathrm{~s}} / \sigma_{\mathrm{Cls}}\right)\right]$, with the element of interest (e.g. $\mathrm{B}$, here) in the numerator, where $\mathrm{I}$ is the intensity (area) of the photoelectron peak and $\sigma$ is the photoionization cross-section for the respective core- level indicated as subscript at a given photon energy (hv) as published by Yeh and Lindau. ${ }^{59}$ The values of $\sigma$ calculated from the reference data ${ }^{\mathrm{i}}$ are: $\sigma_{\mathrm{B} 1 \mathrm{~s}}(\mathrm{~h} v=280 \mathrm{eV})=0.726 ; \sigma_{\mathrm{B} 1 \mathrm{~s}}(\mathrm{~h} v=410 \mathrm{eV})=0.230 ; \sigma_{\mathrm{N} 1 \mathrm{~s}}(\mathrm{~h} v=520 \mathrm{eV})=0.462 ;$ $\sigma_{\mathrm{Cls}}(\mathrm{h} v=410 \mathrm{eV})=0.463 ; \sigma_{\mathrm{Cls}}(\mathrm{h} v=520 \mathrm{eV})=0.312 ; \sigma_{\mathrm{Cls}}(\mathrm{h} v=650 \mathrm{eV})=0.149$

DFT Methods. First principles calculations were performed using Density Functional Theory within the local density approximation using the data fit by Perdew and Zunger ${ }^{60}$ as implemented in the Quantum Espresso package. ${ }^{61}$ Norm conserving pseudopotentials supplied with the package for $\mathrm{B}, \mathrm{C}$, and $\mathrm{N}$ with 3,4 and 5 valence electrons respectively were employed. An energy cut-off of $65 \mathrm{Ry}$ determined the size of the planewave basis set. The different $\mathrm{B} / \mathrm{N}$ dopant configurations are considered in a 7x7 supercell for graphene containing 98 lattice sites. Thus a single $\mathrm{B}$ or $\mathrm{N}$ corresponds to $1 \%$ doping. The atomic structure is allowed to fully relax to minimize the energy within a fixed unit cell determined by the computed minimum energy C-C bond length for graphene (1.41 ̊). Resulting local bond lengths are reported in Figs. S1 and S2. Depending on details of the $\mathrm{B} / \mathrm{N}$ configuration, a modest variation in the bond lengths are found: B-N, 1.40 to $1.45 \AA$; B-C, 1.47 to $1.49 \AA$; N-C, 1.36 to $1.39 \AA$. There is certain amount of internal adjustment within each configuration, including some modest bulging out of plane for B rich configurations (e.g. about $0.3 \AA$ for STM2 where the B-B bond length is $1.54 \AA$ ). The 
overall strain in the supercell is small. The formation energies reported for the B-N complexes are relative to the energy for pristine graphene and isolated graphitic $\mathrm{N}$ and $\mathrm{B}$ dopants, i.e., these reference structures determine the chemical potentials for $\mathrm{C}, \mathrm{N}$ and $\mathrm{B}$ respectively in the calculation of the formation energy of the B-N complexes. The graphene layer was isolated, with no effect of the substrate considered. The STM images were simulated using the TersoffHamann approach. ${ }^{63}$ We recently analyzed the impact of interactions with the $\mathrm{Cu}$ substrate on such simulated STM images and we expect the impact on the present analysis to be minimal. ${ }^{25}$ Naturally, the $\mathrm{Cu}$ substrate is central to the growth mechanism, the details of which are outside the scope of this investigation.

\section{FIGURES}

Figure 1. XPS measured at the B 1s (left), C 1s (center) and N 1s (right) core levels for pristine graphene (PG (a)), co-doped graphene (B/NG (b)) and two $\mathrm{B}_{2} \mathrm{H}_{6}$-grown B-doped graphene samples (BG1 (c), BG2 (d)) shown in solid lines and labeled (a-d) on the left. Dashed curves correspond to peak fitting analysis. C 1s and B 1s data shown here were measured at photon energy of $410 \mathrm{eV}$ and N 1s XPS was measured at $520 \mathrm{eV}$. Atomic B: N percent concentrations based on the data shown here are included for as an inset. 


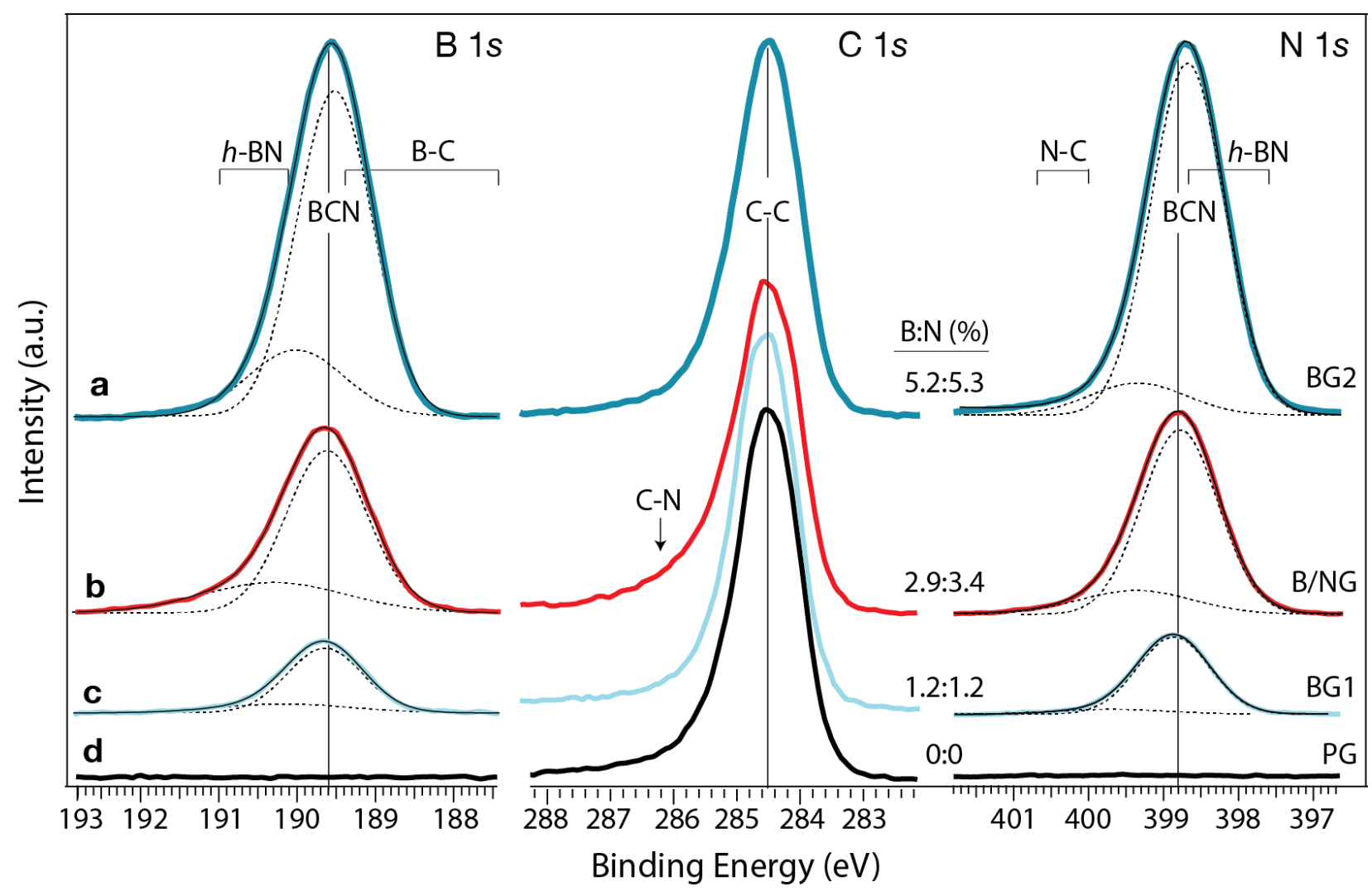

Figure 2. B 1s (left), C 1s (center) and $\mathrm{N}$ 1s (right) incident polarization angle-dependent NEXAFS for (a) BG2, (b) BG1 and (c) B/NG compared to (d) h-BN (left, right) and pristine graphene (PG, center) references. Dashed line $\left(70^{\circ}\right.$ or $\left.90^{\circ}\right)$ and solid line $\left(20^{\circ}\right)$ correspond to measurements with the electric field vector of the incoming beam oriented in- and nearly out-ofplane, respectively, as indicated in the legend and inset. Thin solid lines correspond to NEXAFS 
data measured close to the magic angle of $54^{\circ}$, which represents orientation independent spectra, specifically B 1s data (b-d) and N 1s data for (d).

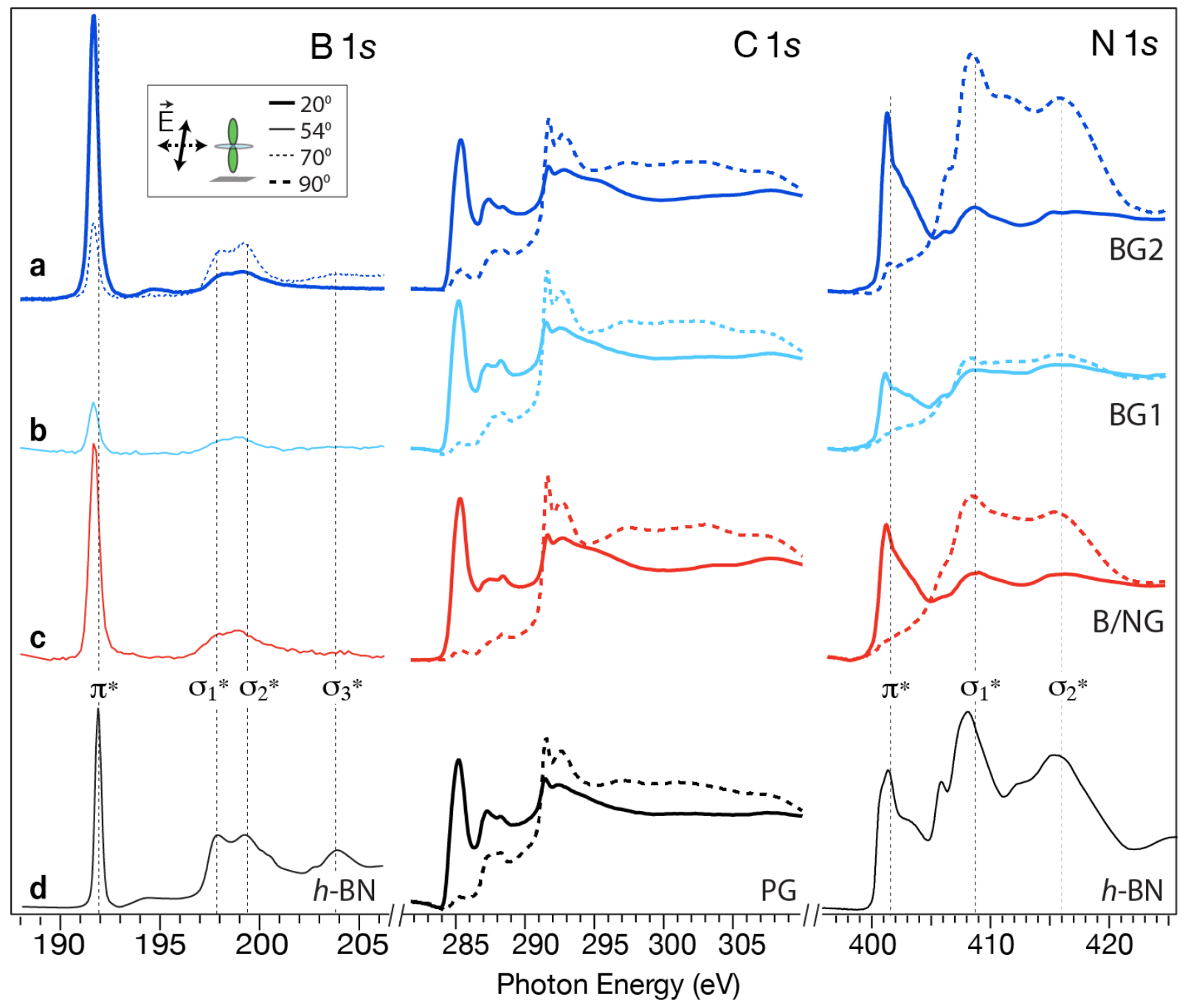

Figure 3. Visualization and identification of individual dopant structures with STM: (a) Experimental STM topography $\left(10 \times 10 \mathrm{~nm}^{2}\right)$ of boron and nitrogen co-doped graphene on copper foil. The triangular features indicated with white and red arrows correspond to individual graphitic nitrogen and boron dopants, respectively. The more complicated features, highlighted in boxes and referred to as STM1 and STM2, respectively are shown over a smaller length scale $\left(1.8 \times 1.4 \mathrm{~nm}^{2}\right)$ in more detail in (b) and (c), respectively, with a potential defect structure for (b) 
STM1; and (c) STM2 overlaid; boron and nitrogen dopant atoms are represented by yellow and green circles, respectively, surrounded by carbon host atoms at the vertices of the overlaid black honeycomb pattern. DFT-based simulated STM patterns for the BNC bond structures describing (d) STM1 and (e) STM2, produce excellent fits to experiment (a-c) and allows atomic-level characterization of $\mathrm{B} / \mathrm{N}$ co-dopant structural motifs in single layer graphene.

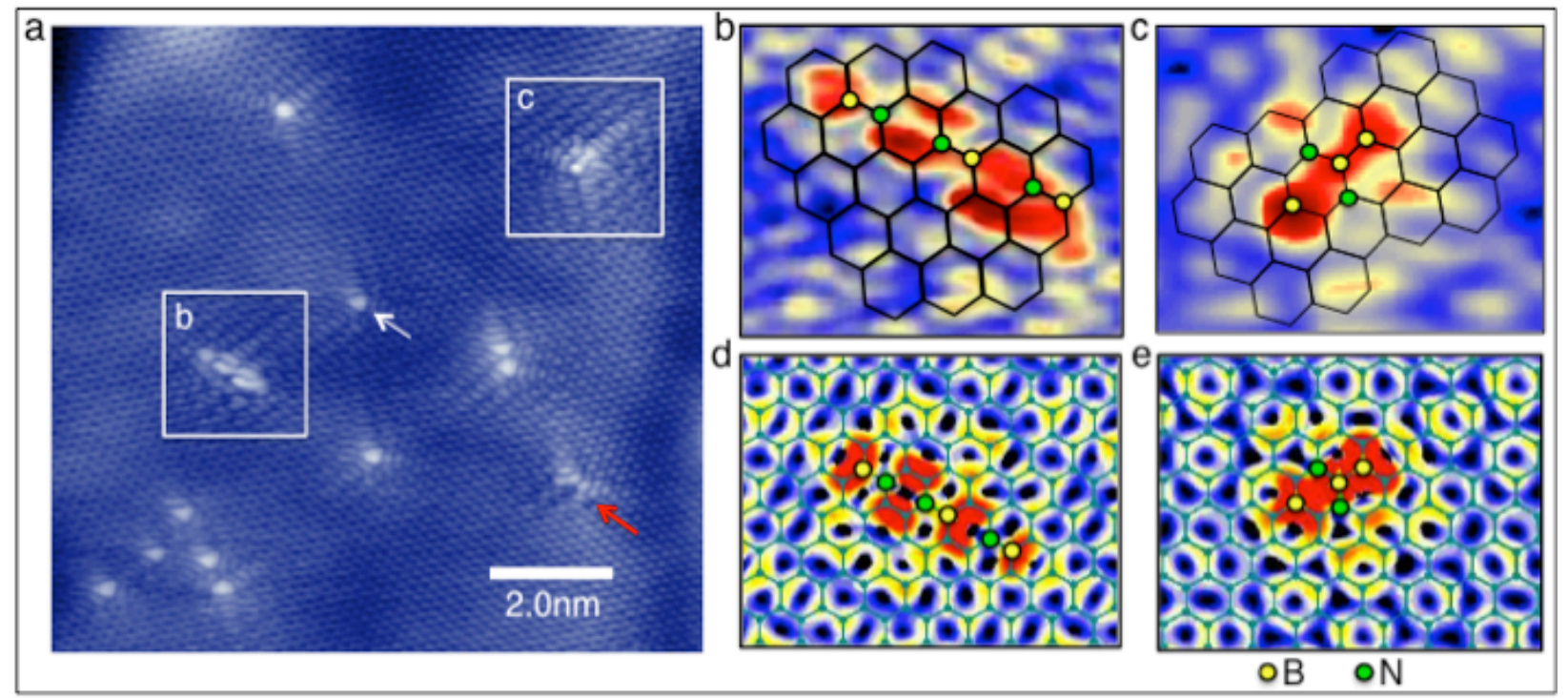

Figure 4. Computed band structure (top) for (a) STM1 and (b) STM2 boron and nitrogen codoped graphene; structures are shown below their respective band diagrams. The Fermi level is highlighted in the thick, solid black line and a small band gap between the valence and conduction bands introduced by the dopants is highlighted with the red circle. Calculations were performed using a 7x7 (98 atom) unit cell for graphene, as described in the theoretical Methods. In this case, the Dirac point once again maps to the $\mathrm{K}$ point of the supercell Brillouin zone. 

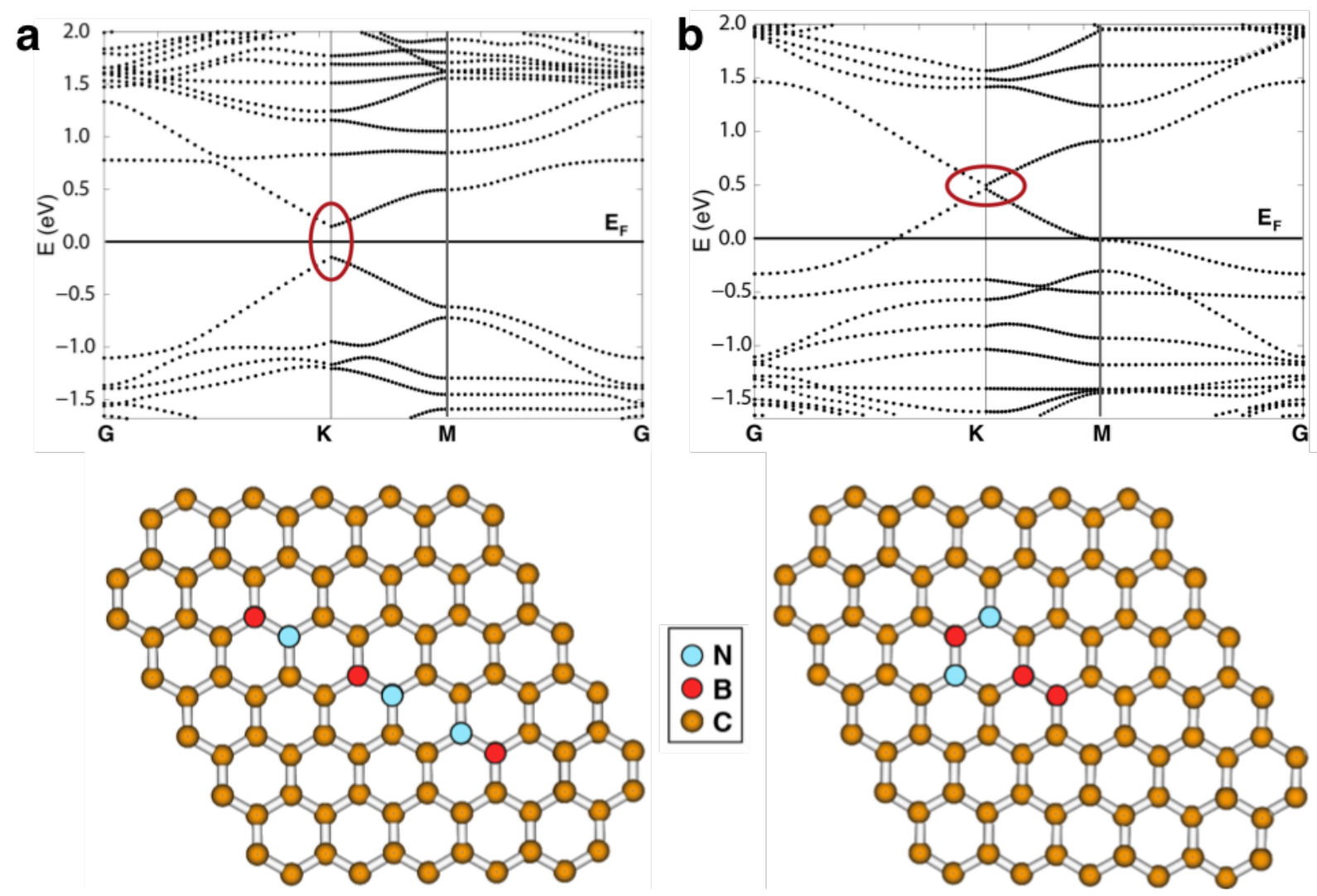

Figure 5. For the structures summarized in Table 1, the computed shift in the work function is plotted versus the net dopant concentration (B minus $\mathrm{N}$ ) in atomic percent (red circles). For comparison, the measured work function shifts (Table 2) are plotted on the same basis for $\mathrm{B} / \mathrm{N}$ co-doped graphene (solid blue squares). Measured and computed work function shifts for nitrogen-doped graphene samples (open blue squares and red circles, respectively) that can incorporate in other motifs that lead to p-type doping have also been added. Measured data are shown from ref. 22 for samples in which a lower concentration of graphitic nitrogen dopants 
(with three bonds to carbon (N3)) than pyridinic nitrogen (two bonds to carbon (N2)) and/or nitrilic (one bond to carbon (N1)) results in a net p-type doping of graphene.

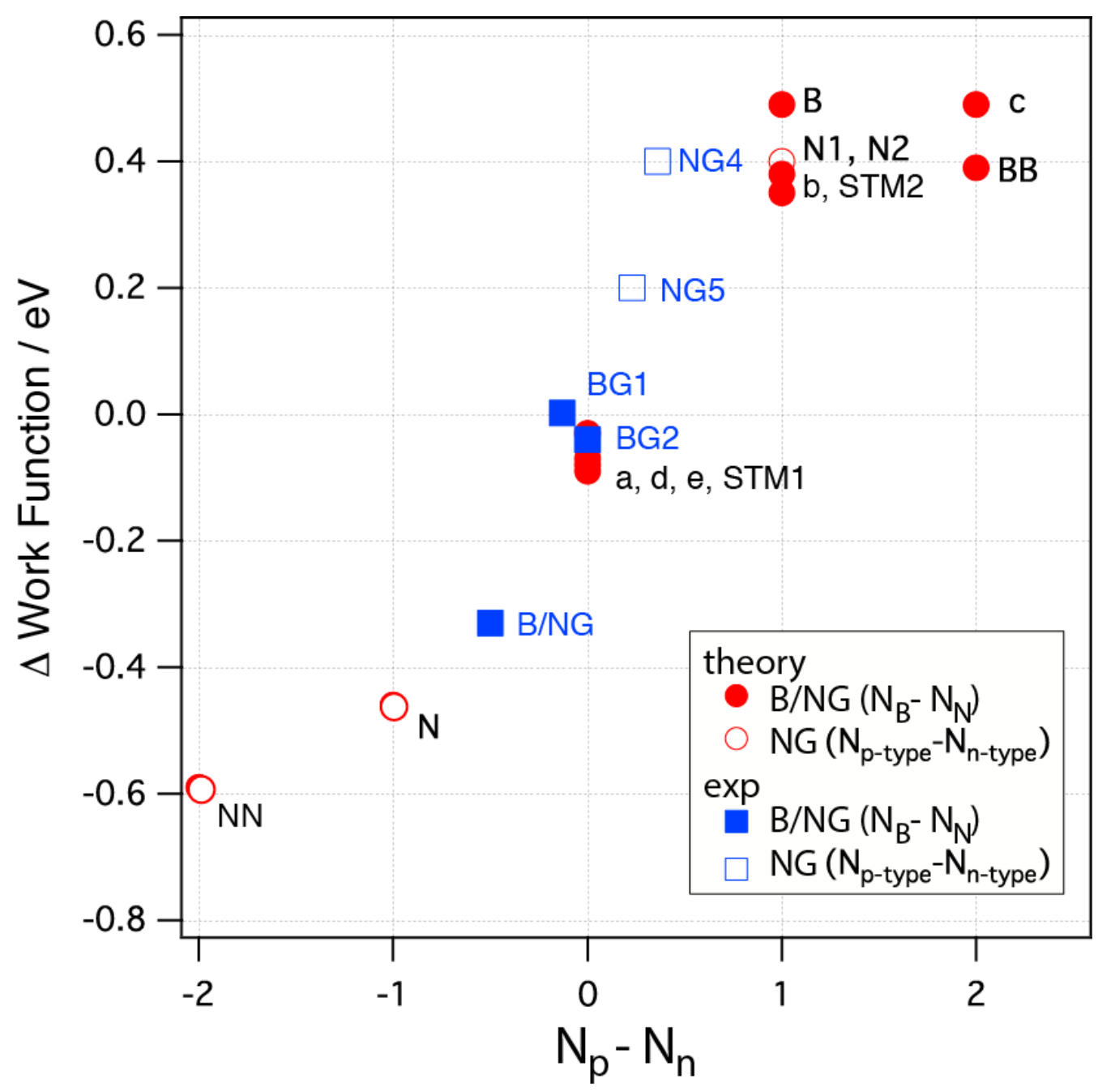

TABLES.

Table 1. Summary of DFT computed properties for a series of dopant structural motifs described in the text and in some cases (labeled a-e) visualized in Fig. S1. Data shown include the number of $\mathrm{B}$ (N_B) and $\mathrm{N}\left(\mathrm{N} \_\mathrm{N}\right)$ substituents in the 98 atom cell, the formation energy (FE), the estimated number of free carriers induced per net number of dopants (n/(N_B-N_N)), the shift in the Dirac point energy (taken a the midpoint if a gap has opened) from the Fermi level (EF) and the shift of the work function ( $\Delta$ W.F.), relative to that calculated for pristine graphene. 


\begin{tabular}{|c|c|c|c|c|c|c|}
\hline & N_B & N_N & FE & n/(N_B-N_N) & E-Dirac-EF & $\Delta$ W.F. \\
\hline B & 1 & 0 & 0.00 & 0.57 & 0.56 & 0.49 \\
\hline $\mathrm{BB}$ & 2 & 0 & 1.21 & 0.38 & 0.64 & 0.39 \\
\hline $\mathrm{N}$ & 0 & 1 & 0.00 & 0.54 & -0.54 & -0.46 \\
\hline $\mathrm{NN}$ & 0 & 2 & 1.20 & 0.53 & -0.75 & -0.59 \\
\hline $\mathrm{BN}(\mathrm{a})$ & 1 & 1 & -2.35 & - & 0.00 & -0.07 \\
\hline STM2 & 3 & 2 & -4.13 & 0.44 & 0.49 & 0.35 \\
\hline STM1 & 3 & 3 & -7.16 & - & 0.00 & -0.03 \\
\hline NBB (b) & 2 & 1 & -3.64 & 0.54 & 0.54 & 0.38 \\
\hline NBBB (c) & 3 & 1 & -4.36 & 0.47 & 0.71 & 0.49 \\
\hline BNBN1 (d) & 2 & 2 & -5.77 & - & 0.00 & -0.08 \\
\hline BNBN2 (e) & 2 & 2 & -5.87 & - & 0.00 & -0.09 \\
\hline
\end{tabular}

Table 2. Atomic boron: carbon: nitrogen (B: C: N) ratios and measured work function shift ( $\Delta$ w.f.) relative to pristine graphene (PG) obtained from XPS and photoemission threshold experiments, respectively, for boron and nitrogen co-doped graphene (corresponding x-ray spectroscopy measurements at each elemental K-edge shown in Fig. 1 (XPS) and Fig. 2 (NEXAFS).

\begin{tabular}{|ccc|}
\hline Sample & B: C: N (\%) & $\Delta$ W.F. (eV) \\
\hline PG & $0.0: 100: 0.0$ & 0.000 \\
B/NG & $2.9: 93.7: 3.4$ & -0.330 \\
\hline
\end{tabular}




\begin{tabular}{|lll|}
\hline BG1 & $1.2: 97.6: 1.2$ & -0.040 \\
BG2 & 5.1: 89.7: 5.2 & 0.002 \\
\hline
\end{tabular}

\section{ASSOCIATED CONTENT}

Supporting Information. The contents of the Supporting Information include: additional B/N co-dopant configurations in for which results appear in Table 1 (Fig. S1); Correlation between computed work function shifts and Dirac point energy shifts for the structures reported in Table 1 (Fig. S2); additional $\mathrm{N} 1 \mathrm{~s}$ and $\mathrm{C} \underline{1 \mathrm{~s}}$ XPS data for Nitrogen-doped $\left(\mathrm{NH}_{3}\right.$-grown, $\left.\mathrm{CVD}\right)$ graphene used to obtain atomic nitrogen concentrations (Fig. S3); Peak fitting of the experimental N 1s XPS data to identify relative concentrations of different nitrogen species in the $\mathrm{N}$-doped graphene (Fig. S4); and details of the peak fitting used to correlate doping level with work function shifts based on measurements of p-type graphene (Table S1) shown in Fig. 5 of the main text. This material is available free of charge via the Internet at http://pubs.acs.org.

\section{AUTHOR INFORMATION}

\section{Corresponding Author}

*email: ts2526@columbia.edu

\section{AUTHOR CONTRIBUTIONS}

The manuscript was written through contributions of all authors. All authors have given approval to the final version of the manuscript.

\section{ACKNOWLEDGMENT}


Research supported by the NSF MRSEC program through Columbia in the Center for Precision Assembly of Superstratic and Superatomic Solids (DMR-1420634). NEXAFS, XPS and photoemission threshold (work function) experiments were performed at beamline 10-1 at the Stanford Synchrotron Radiation Laboratory, a national user facility operated by Stanford University on behalf of the U.S. Department of Energy, Office of Basic Energy Sciences (TS, DN). Additional NEXAFS experiments were also carried out at beamline U7A at the National Synchrotron Light Source and theory and computations were carried out in part at the Center for Functional Nanomaterials, both of which are U.S. DOE Office of Science Facilities, at Brookhaven National Laboratory; under Contract No. DE-SC0012704 (MH, CJ, TS). STM experiments were supported by the Office of Naval Research (award number N00014-14-10501) and by the Air Force Office of Scientific Research (AFOSR) (award number FA9550-111-0010) (LZ, ANP). Theoretical calculations were performed by LP, DR, and MH. Sample growth was performed by ML and JP.

\section{ABBREVIATIONS}

h-BN hexagaonal boron nitride; STM Scanning Tunneling Microscopy; LT-STM Low temperature scanning tunneling microscopy; DFT Density Functional Theory; B (boron); N (nitrogen); XPS X-ray Photoelectron Spectroscopy; NEXAFS Near Edge X-ray Absorption Fine Structure; B/N boron/nitrogen; CVD Chemical Vapor Deposition; BNC boron nitride carbide; PG pristine graphene; B/NG boron and nitrogen co-doped graphene; BG boron-doped graphee,, BG1, BG2 $\mathrm{B}_{2} \mathrm{H}_{6}$-grown graphene samples; UHV ultrahigh-vaccum; C 1s Carbon K-edge; $\mathrm{N}$ 1s Nitrogen K-edge; B 1s Boron K-edge; FWHM full width at half maximum; FE formation 
energy; N_B (N_N) number of B (N) substituents in the 98 atom cell; EF Fermi level; W.F. work function.

\section{REFERENCES}

1. Nair, R. R.; Blake, P.; Grigorenko, A. N.; Novoselov, K. S.; Booth, T. J.; Stauber, T.; Peres, N. M. R.; Geim, A. K. Fine Structure Constant Defines Visual Transparency of Graphene. Science 2008, 320, 1308.

2. Ci, L.; Song, L.; Jin, C.; Jariwala, D.;Wu, D.; $\quad$ Li, Y.; Srivastava, A.; Wang, Z. F.; Storr, K.; Balicas, L.; Liu, F.; Ajayan, P.M. Atomic layers of hybridized boron nitride and graphene domains. Nat. Mater. 2010, 9, 430-435.

3. van der Zande A. M.; Huang, P. Y.; Chenet, D. A.; Berkelbach, T. C.; You, Y.; Lee, G.H.; Heinz, T. F.; Reichman, D. R.; Muller, D. A.; Hone, J. C. Grains and grain boundaries in highly crystalline monolayer molybdenum disulphide. Nature Mater. 2013, 12, 554-561.

4. Lee, C.-H.; Lee, G.-H., van der Zande, A. M.; Chen, W.; Li, Y.; Han, M.; Cui, X.; Arefe, G.; Nuckolls, C.; Heinz, T. F.; Guo, J.; Hone, J.C.; Kim, P.; Atomically thin p-n junctions with van der Waals heterointerfaces Nature Nanotech. 2014, 9, 676-681.

5. Geim, A. K.; Grigorieva, I. V. Van der Waals heterostructures. Nature 2013, 499, 419425. 
6. Song, L.; Liu, Z.; Reddy, A. L. M.; Narayanan, N. T.; Taha-Tijerina, J.; Peng, J.; Gao, G.; Lou, J.; Vajtai, R. and Ajayan, P. M. Binary and Ternary Atomic Layers Built from Carbon, Boron, and Nitrogen. Adv. Mater. 2012, 24, 4878-4895.

7. Cox, M.; Gorodetsky, A.; Kim, B.; Kim, K. S.; Jia, Z.; Kim, P.; Nuckolls, C.; Kymissis, I. Single-layer graphene cathodes for organic photovoltaics. App. Phys. Lett. 2011, 98, 123303.

8. Ozaki, J; Anahara, T.; Kimura, N.; Oya, A. Simultaneous doping of boron and nitrogen into a carbon to enhance its oxygen reduction activity in proton exchange membrane fuel cells. Carbon 2006, 44, 3358-3361.

9. Wang, S.; Iyyamperumal, E.; Roy, A.; Xue, Y.; Yu, D.; Dai, L. M. Vertically aligned $\mathrm{BCN}$ nanotubes as efficient metal-free electrocatalysts for the oxygen reduction reaction: A synergetic effect by co-doping with boron and nitrogen. Angew. Chem. Int. Ed. 2011, 50, 1175611760.

10. Wu, Z. S.; Ren, W. C.; Xu, L.; Li, F.; Cheng, H. M. Doped Graphene Sheets As Anode Materials with Superhigh Rate and Large Capacity for Lithium Ion Batteries, ACS Nano 2011, 5, 5463-5471.

11. Jeong, H. M.; Lee, J. W.; Shin, W. H.; Choi, Y. J.; Shin, H. J.; Kang, J. K.; Choi, J. W. Nitrogen-doped graphene for high-performance ultracapacitors and the importance of nitrogendoped sites at basal planes. Nano Lett. 2011, 11, 2472-2477.

12. Wu, Z. -S.; Winter, A.; Chen, L.; Sun, Y.; Turchanin, A.; Feng, X.; Müllen, K. ThreeDimensional Nitrogen and Boron Co-doped Graphene for High-Performance All-Solid-State Supercapacitors. Adv. Mater. 2012, 24, 5130-5135. 
13. Wang, H; Maiyalagan, T.; Wang, X. Review on recent progress in nitrogen-doped graphene: synthesis, characterization, and its potential applications. ACS Catal. 2012, 2, 781794.

14. Lee, C.-H.; Schiros, T.; Santos, E. J. G.; Kim, B.; Yager, K.G.; Kang, S.J.; Lee, S.; Yu, J.; Watanabe, K.; Taniguchi, T.; Hone, J.C.; Kaxiras, E.; Nuckolls, C.; Kim, P. Epitaxial Growth of Molecular Crystals on van der Waals Substrates for High-Performance Organic Electronics. Adv. Mat. 2014, 26, 2812-2817.

15. Ohta, T.; Bostwick, A.; Seyller, T.; Horn, K.; Rotenburg,E. Controlling the Electronic Structure of Bilayer Graphene. Science 2006, 313, 951-954.

16. Li, X.; Wang, H.; Robinson, J. T.; Sanchez, H.; Diankov, G.; Dai,H. Simultaneous Nitrogen Doping and Reduction of Graphene Oxide. J. Am. Chem. Soc. 2009, 131, 15939-15944.

17. Liu, H.; Liu, Y.; Zhua, D. Chemical Doping of Graphene. J. Mater. Chem. 2011, 21, $3335-3345$.

18. Guo, B.; Liu, Q.; Chen, E.; Zhu, H.; Fang, L.; Gong, J. R. Controllable N-doping of graphene. Nano Lett. 2010, 10, 4975-4980.

19. Wang, X.; Li, X.; Zhang, L.; Yoon, Y.; Weber, P.; Wang, H.; Guo,J.; Dai, H. N-doping of graphene through electrothermal reactions with ammonia. Science 2009, 324, 768-771.

20. Wang, Y.; Shao, Y.; Matson, D. W.; Li, U.; Lin, Y. Nitrogen-Doped Graphene and Its Application in Electrochemical Biosensing. ACS Nano 2010, 4, 1790-1798. 
21. Zhao, L. ; He, R. K.; Rim, T.; Schiros, T.; Kim, K. S.; Zhou, H.; Gutiérrez, C.; Chockalingam, S. P.; Arguello, C.J.; Pálová, L.; Nordlund, D.; Hybertsen, M. S.; Reichman, D.R.; Heinz, T.F.; Kim, P.; Pinczuk, A.; Flynn G.W.; Pasupathy, A.N. Visualizing Individual Nitrogen Dopants in Monolayer Graphene. Science 2011, 333, 999-1003.

22. Schiros, T.; Nordlund, D.; Pálová, L.; Prezzi, D.; Zhao, L.; Kim, K. S.; Wurstbauer, U.; Gutiérrez, C.; Delongchamp, D.; Jaye, C.; Fischer, D.; Ogasawara, H.; Pettersson, L. G. M.; Reichman, D. R.; Kim, P.; Hybertsen, M. S.; Pasupathy, A. N. Connecting dopant bond type with electronic structure in N-doped graphene. Nano Lett. 2012, 12, 4025-4031.

23. Gebhardt, J.; Koch, R. J.; Zhao, W.; Höfert, O.; Gotterbarm, K.; Mammadov, S.; Papp, C.; Görling, A.; Steinruck; H. -P.; Seyller, Th. Growth and electronic structure of boron-doped graphene. Phys. Rev. B 2013, 87, 155437.

24. Kim, Y. A.; Fujisawa, K.; Muramatsu, H.; Hayashi, T.; Endo, M.; Fujimori, T.; Kaneko, K.; Terrones, M.; Behrends, J.; Eckmann, A.; Casiraghi, C.; Novoselov, K. S.; Saito, R.; Dresselhaus. M. S. Raman spectroscopy of boron-doped single-layer graphene. ACS Nano 2012, 7, 6293-6300.

25. Zhao, L.; Levendorf, M. P.; Goncher, S. J.; Schiros, T.; Pálová, L.; Zabet-Khosousill, A.; Taeg Rim, K.; Gutiérréz, C.; Nordlund, D.; Jaye, C.; Hybertsen, M.; Reichman, D.; Flynn, G.W.; Park, J.; Pasupathy, A. N. Local atomic and electronic structure of boron chemical doping in monolayer graphene. Nano Lett. 2013, 13, 4659-4665.

26. Zhang, X.; Schiros, T.; Nordlund, D.; Shin, Y. C.; Kong, J.; Dresselhaus, M.; Palacios, T. X-ray Spectroscopic Investigation of Chlorinated Graphene: Surface Structure and Electronic Effects. Adv. Funct. Mater. 2015, 25, 4163-4169. 
27. Zhang, X.; Hsu, A.; Wang, H.; Song, Y.; Kong, J.; Dresselhaus, M.S.; Palacios, T. Impact of Chlorine Functionalization on High-Mobility Chemical Vapor Deposition Grown Graphene. ACS Nano 2013, 7, 7262-7270.

28. Tison, Y.; Lagoute, J.; Repain, V.; Chacon, C.; Girard, Y.; Rousset, S.; Joucken, F.; Sharma, D.; Henrard, L.; Amara, H.; Ghedjatti, A.; Ducastelle, F. Electronic Interaction between Nitrogen Atoms in Doped Graphene. ACS Nano 2015, 9, 670-678.

29. Lambin, P.; Amara, H.; Ducastelle, F.; Henrard, L. Long-range interactions between substitutional nitrogen dopants in graphene: Electronic properties calculations. Phys. Rev. B $2012,86,045448$.

30. Rani, P.; Jindal, V. K. Designing band gap of graphene by B and N dopant atoms. RSC Adv. 2013,3,802.

31. Stephan O.; Ajayan, P. M.; Colliex C.; Redlich P.; Lambert, J. M.; Bernier P.; Lefin P. Doping graphitic and carbon nanotube structures with boron and nitrogen. Science 1994, 266, $1683-1685$.

32. Menezes, M. G.; Capaz, R. B. Half-metallicity induced by charge injection in hexagonal boron nitride clusters embedded in graphene. Phys. Rev. B 2012, 86, 195413.

33. Chang, C. -K.; Kataria, S.; Kuo, C. -C.; Ganguly, A.; Wang, B. -Y.; Hwang, J. -Y.; Huang, K .-J.; Yang, W. -H.; Wang, S. -B.; Chuang, C. -H.; Chen, M.; Huang, C. -I.; Pong, W. F.; Song, K. -J.; Chang, S. -J.; Guo, J. -H.; Tai, Y.; Tsujimoto, M.; Isoda, S.; Chen, C. -W.; Chen, L. -C.; Chen, K. -H. Band Gap Engineering of Chemical Vapor Deposited Graphene by in Situ BN Doping. ACS Nano 2012, 7, 1333-1341. 
34. Zhao, R.; Wang, J.; Yang, M.; Liu, Z.; Liu, Z. BN-Embedded Graphene with a Ubiquitous Gap Opening. J. Phys. Chem. C 2012, 116, 21098.

35. Deng, X.;Wu, Y.; Dai, J.; Kang, D.; Zhang, D. Electronic structure tuning and band gap opening of graphene by hole/electron codoping. Physics Letters A 2011, 375, 3890-3894.

36. Ferrighi, F.; Trioni, M. I.; Di Valentin, C. Boron-Doped, Nitrogen-Doped, and Codoped Graphene on Cu(111): A DFT + vdW Study. J. Phys. Chem. C 2015, 119, 6056.

37. Rani, P.; Dubey, G. S.; Jindal, V.K. DFT study of optical properties of pure and doped graphene. Physica E 2014, 62, 28-35.

38. Yuea, J.; Chenga, W.; Zhanga, X.; Hea, D.; Chen, G. Ternary BCN thin films deposited by reactive sputtering. Thin Solid Films 2000, 375, 247-250.

39. Morant, C.; Prieto, P.; Bareño, J.; Sanz, J.M.; Elizalde, E. Hard $\mathrm{BC}_{\mathrm{x}} \mathrm{N}_{\mathrm{y}}$ thin films grown by dual ion beam sputtering. Thin Solid Films 2006, 515, 207-211.

40. Watanabe; M. O.; Itoh, S.K.; Mizushima, K.: Sasaki, T. Bonding characterization of $\mathrm{BC}_{2} \mathrm{~N}$ thin films. Appl. Phys. Lett. 1996, 68, 2962.

41. Zheng, W.T.; Xinga, K.Z.; Hellgrena, N.; Lögdlunda, M.; Johanssona, Å.; Gelivsb, U.; Salanecka, W.R.; Sundgrena, J.-E. Nitrogen 1s electron binding energy assignment in carbon nitride thin films with different structures. J. El. Spec. Rel. Phen. 1997, 87, 45-49.

42. Hellgren, N.; Guo, J.; Luo, Y.; Sathe, C.; Agui, A.; Kashtanov, S.;Nordgren, J.; Ågren, H.; Sundgren, J.-E. Nitrogen bonding structure in carbon nitride thin films studied by soft x-ray spectroscopy. App. Phys. Lett. 2001, 79, 4348-50. 
43. Abdul Mannan, Md.; Noguchi, H.; Kida, T.; Nagano, M.; Hirao, N.; Baba, Y. Chemical bonding states and local structures of the oriented hexagonal BCN films synthesized by microwave plasma CVD. Mater. Sci. Semicond. Process. 2008, 11, 100-105.

44. Abdul Mannan, Md.; Noguchi, H.; Kida, T.; Nagano, M.; Hirao, N.; Baba, Y. Growth and characterization of stoichiometric $\mathrm{BCN}$ films on highly oriented pyrolytic graphite by radiofrequency plasma enhanced chemical vapor deposition. Thin Solid Films 2010, 518, 41634169.

45. Bake, O.; Hoffmann, P. S.; Klein, A; Pollakowski, B; Beckhoff, B; Kosinova, M. L.; Fainer, N. I.; Sulyaeva, V. S.; Trunova, V. A.; Ensinger, W. Speciation of $\mathrm{BC}_{\mathrm{x}} \mathrm{N}_{\mathrm{y}}$ films grown by PECVD with trimethylborazine precursor. Anal Bioanal Chem 2009, 395, 1901-1909.

46. Mannan, M. A.; Baba, Y.; Kida, T.; Nagano, M.; Shimoyama, I.; Hirao, N.; Noguchi, H. Orientation of B-C-N Hybrid Films Deposited on Ni (111) and Polycrystalline Ti Substrates Explored by X-Ray Absorption Spectroscopy. Thin Solid Films 2011, 519, 1780- 1786.

47. Shi, Y.; Hamsen, C.; Jia, X.; Kim, K. K.; Reina, A.; Hofmann, M.; Hsu, A. L.; Zhang, K.; Li, H.; Juang. Z.-Y.; Dresselhaus, M. S.; Li, L.-J.; Kong, J. Synthesis of Few-Layer Hexagonal Boron Nitride Thin Film by Chemical Vapor Deposition. Nano Lett. 2010, 10 , 4134-4139.

48. Lee, K.S.; Kima, Y.S.; Tosab, M.; Kasaharab, A.; Yosiharab, K. Mechanical properties of hexagonal boron nitride synthesized from film of $\mathrm{Cu} / \mathrm{BN}$ mixture by surface segregation. App . Surf. Sci. 2001, 169, 420-424. 
49. Jacobsohn, L.G.; Schulze, R.K.: Maia da Costa, M.E.H.; Nastasi, M. X-ray photoelectron spectroscopy investigation of boron carbide films deposited by sputtering. Surface Science 2004, $572,418-424$.

50. Jiménez, I.; Sutherland, D. G. J.; van Buuren, T.; Carlisle, J. A.; Terminello, L. J.; Himpsel, F. J. Photoemission and x-ray-absorption study of boron carbide and its surface thermal stability. Phys. Rev. B 1998, 57, 13167.

51. J. Stohr. NEXAFS Spectroscopy. Springer-Verlag (1992).

52. Bruhwiler, P. A; Maxwell, A. J.; Puglia, C; Nilsson, A.; Andersson, S.; Mårtensson, N. $\pi^{*}$ and $\sigma^{*}$ Excitons in C 1s Absorption of Graphite. Phys. Rev. Lett. 1995, 74, 614-617.

53. Al-Aqtash, K. M.; Al-Tarawneh, T.; Tawalbeh, T.; Vasiliev, I. Ab initio study of the interactions between boron and nitrogen dopants in graphene. J. Appl. Phys. 2012, 112, 034304.

54. Moscovici, J.; Loupias, G.: Parent, P.H.; Tourillo, G. Polarization-dependent boron and nitrogen K NEXAFS of hexagonal BN. J. Phys. Chem. Solids 1996, 57, 1159-1161.

55. Bozanica, A.; Petravica, M.; Fanb, L.-J.; Yangb, Y.-W.; Chen, Y. Direct observation of defect levels in hexagonal BN by soft X-ray absorption spectroscopy. Chem. Phys. Lett. 2009, 472, 190-193.

56. Uddin, Md. N.; Shimoyama, I.; Baba, Y.; Sekiguchi, T.; Nagano, M. X-ray photoelectron spectroscopic observation on B-C-N hybrids synthesized by ion beam deposition of borazine. $J$. Vac. Sci. Technol. A 2005, 23, 497. 
57. Yuge, K. Phase stability of boron carbon nitride in a heterographene structure: A firstprinciples study. Phys. Rev. B 2009, 79, 144109.

58. Krsmanović, R. S.; Šljivančanin, Ž. Atomic Structure, Electronic Properties, and Reactivity of In-Plane Heterostructures of Graphene and Hexagonal Boron Nitride. J. Phys. Chem. C 2014, 118, 16104.

59. (a) Yeh, J.-J.; Lindau, I. Atomic Subshell Photoionization Cross Sections and Asymmetry Parameters: $1<\mathrm{Z}<$ 103. At. Data Nucl. Data Tables 1985, 32, 1. (b) Yeh, J.-J.. Atomic Calculations of Photoionization Cross Sections and Asymmetry Parameters; Gordon and Breach: Langhorne, PA, 1993.

60. Perdew, J.P.; Zunger; A. Self-interaction correction to density-functional approximations for many-electron systems. Phys. Rev. B 1981, 23, 5048.

61. Giannozzi, P. et al. QUANTUM ESPRESSO: a modular and open-source software project for quantum simulations of materials. J. Phys. Condens. Matter 2009, 21, 395502.

TOC figure: Scanning Tunneling Microscopy images, combined with atom-specific x-ray spectroscopy and density functional theory, reveal an atomistic picture of local boron/nitrogen co-dopant bond structures in monolayer graphene and their electronic structure effects. 


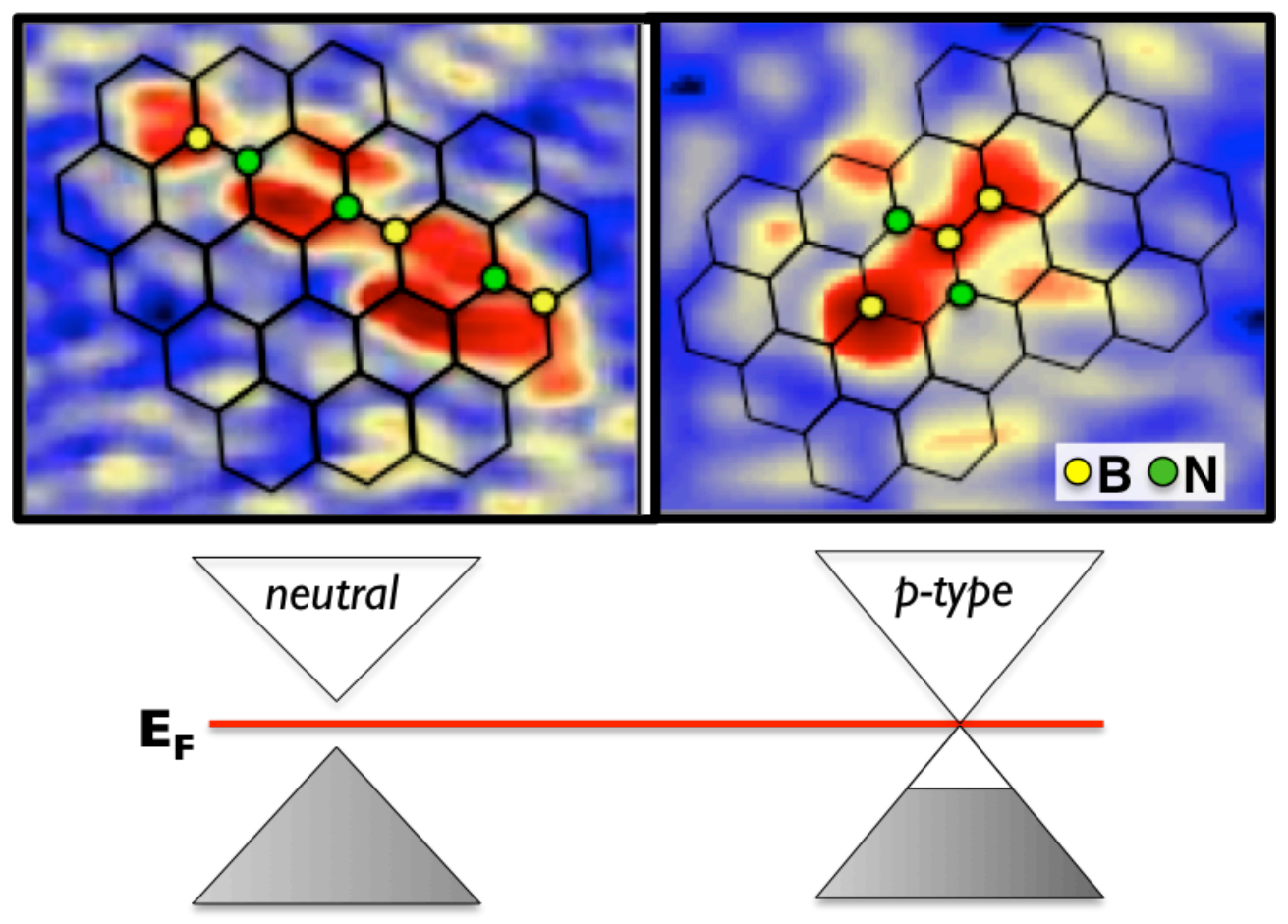




\section{Atomistic Interrogation Of B-N Co-Dopant}

\section{Structures And Their Electronic Effects In}

\section{Graphene}

Theanne Schiros ${ }^{1 a, b *}$, Dennis Nordlund ${ }^{2}$, Lucia Palova ${ }^{3}$, Liuyan Zhao ${ }^{4}$, Mark Levendorf ${ }^{5}$, Cherno Jaye ${ }^{6}$, David Reichman ${ }^{3}$, Jiwong Park ${ }^{5}$, Mark Hybertsen $^{7}$, Abhay Pasupathy ${ }^{4}$

${ }^{1 a}$ MRSEC, Columbia University, New York, NY 10027, USA; ${ }^{1 b}$ Fashion Institute of Technology/State University of New York, Department of Science and Mathematics, New York, NY, 10001, USA.

${ }^{2}$ Stanford Synchrotron Radiation Lightsource, SLAC National Accelerator Laboratory, Menlo Park, CA 94025, USA

${ }^{3}$ Department of Chemistry, Columbia University, New York, NY 10027, USA

${ }^{4}$ Department of Physics, Columbia University, New York, NY 10027, USA

${ }^{5}$ Chemistry Department, Cornell University, Ithaca, New York 10065, USA

${ }^{6}$ Materials Measurement Laboratory, National Institute of Standards and Technology, Gaithersburg, Maryland 20899,USA

${ }^{7}$ Center for Functional Nanomaterials, Brookhaven National Laboratory, Upton, New York 11973, USA 


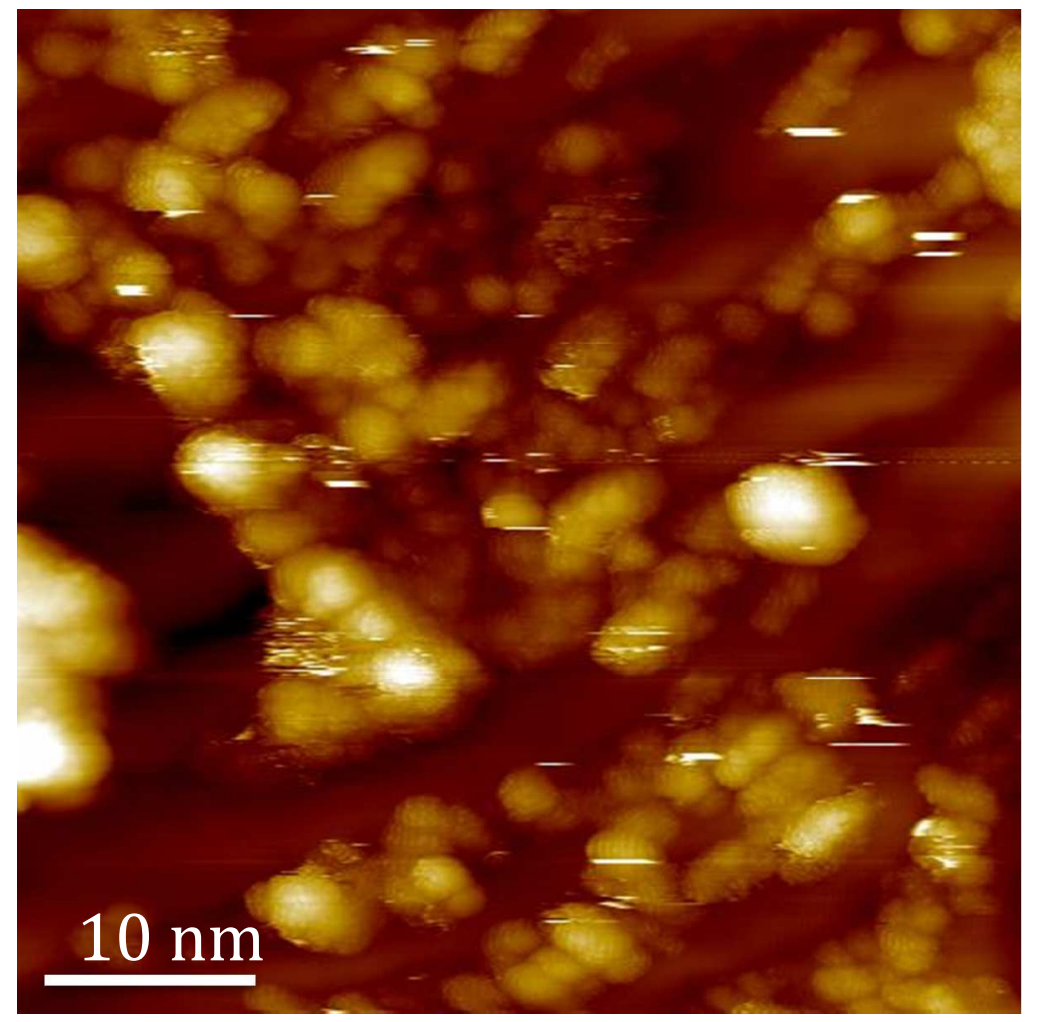

Figure S1. Example of a rough graphene film created during doped graphene growth. The height variation across the substrate is several tens of nanometers, which makes atomic-resolution imaging impossible. Codoped samples suffer most severely from this problem, and flat, clean areas are impossible to identify on these samples. 


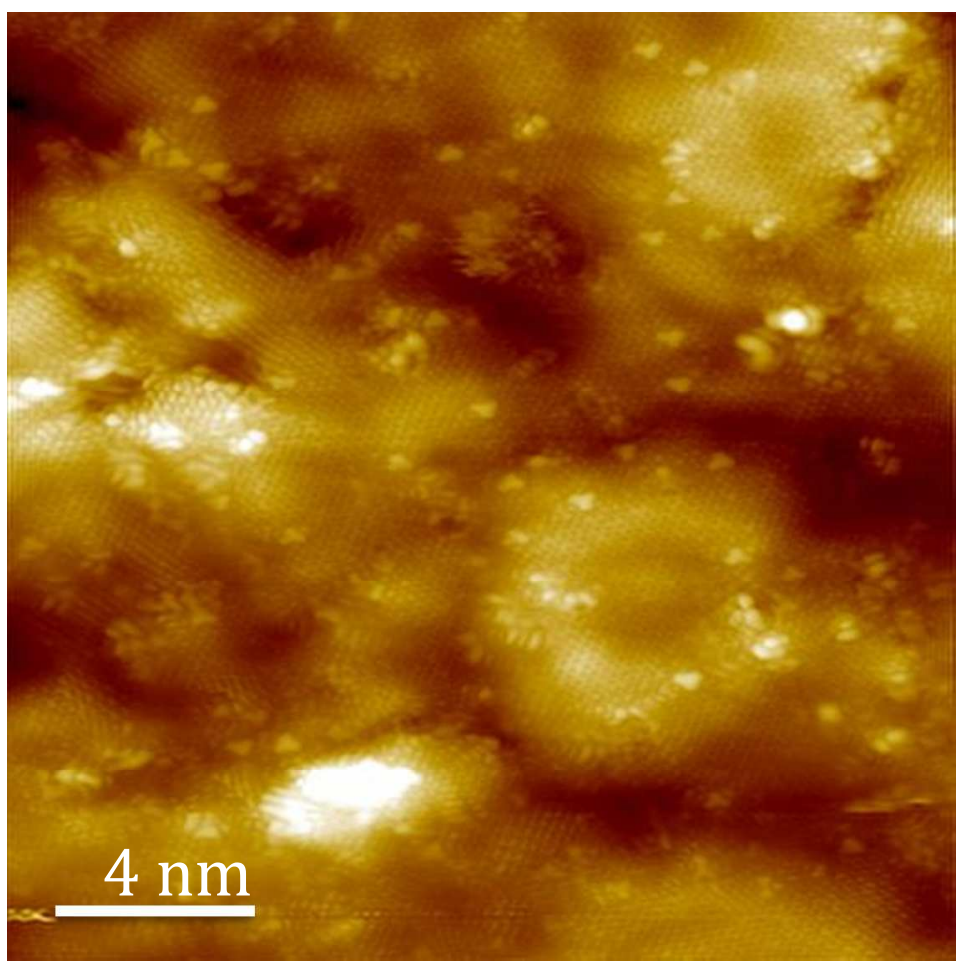

Figure S2. Large area topograph of sample BG2 showing the presence of graphitic boron, dopant configurations STM1 and STM2, and other more complex dopant features.

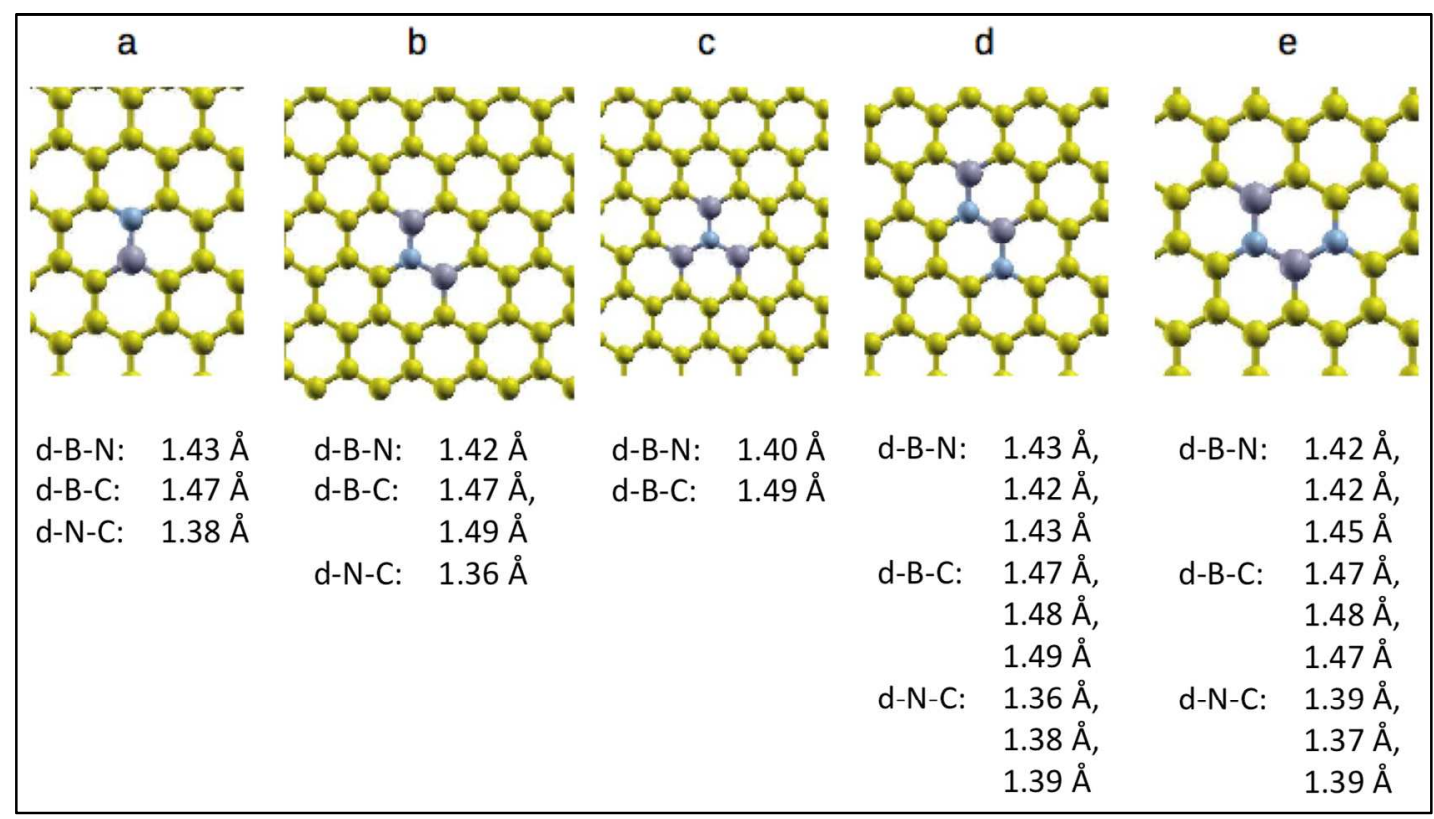

Figure S3. Additional B/N co-dopant configurations in graphene (carbon atoms: yellow; nitrogen atoms: purple; and boron atoms: light blue) for which DFT calculations were performed and for which results appear in Table 1. Symmetry distinct bond lengths near the B/N cluster, from the relaxed structures based on DFT, are listed below in the order in which they appear moving from upper left to lower right. 

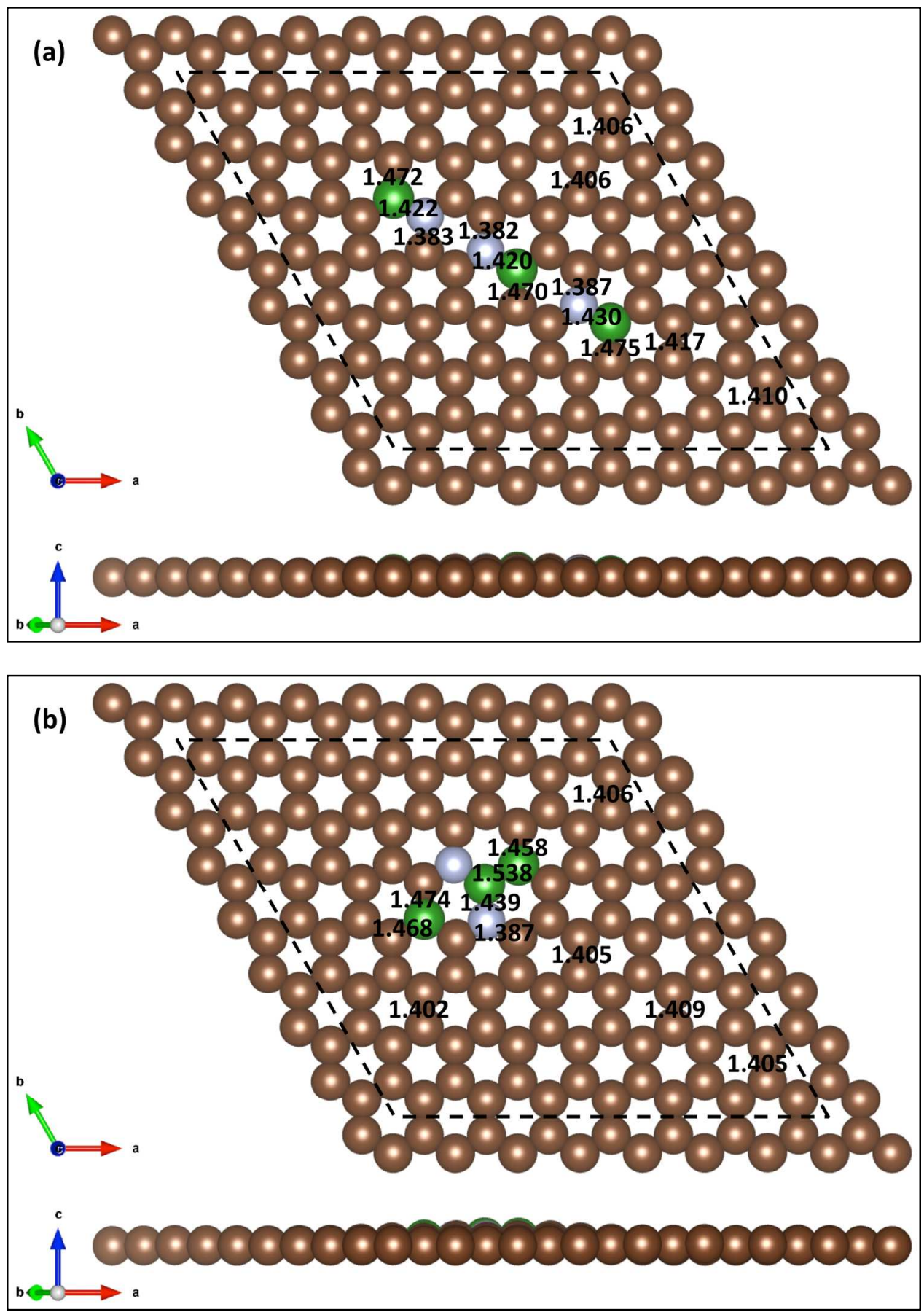

Figure S4. Visualization of the structures discussed in the text as (a) STM1 and (b) STM2. A top view and a side view are shown with a space filling representation created using VESTA ${ }^{1}$.(C atoms brown, $\mathrm{N}$ atoms blue, $\mathrm{B}$ atoms green). The $7 \times 7$ supercell is indicated by the dashed lines. Selected local bond lengths are superposed in units of angstrom. 


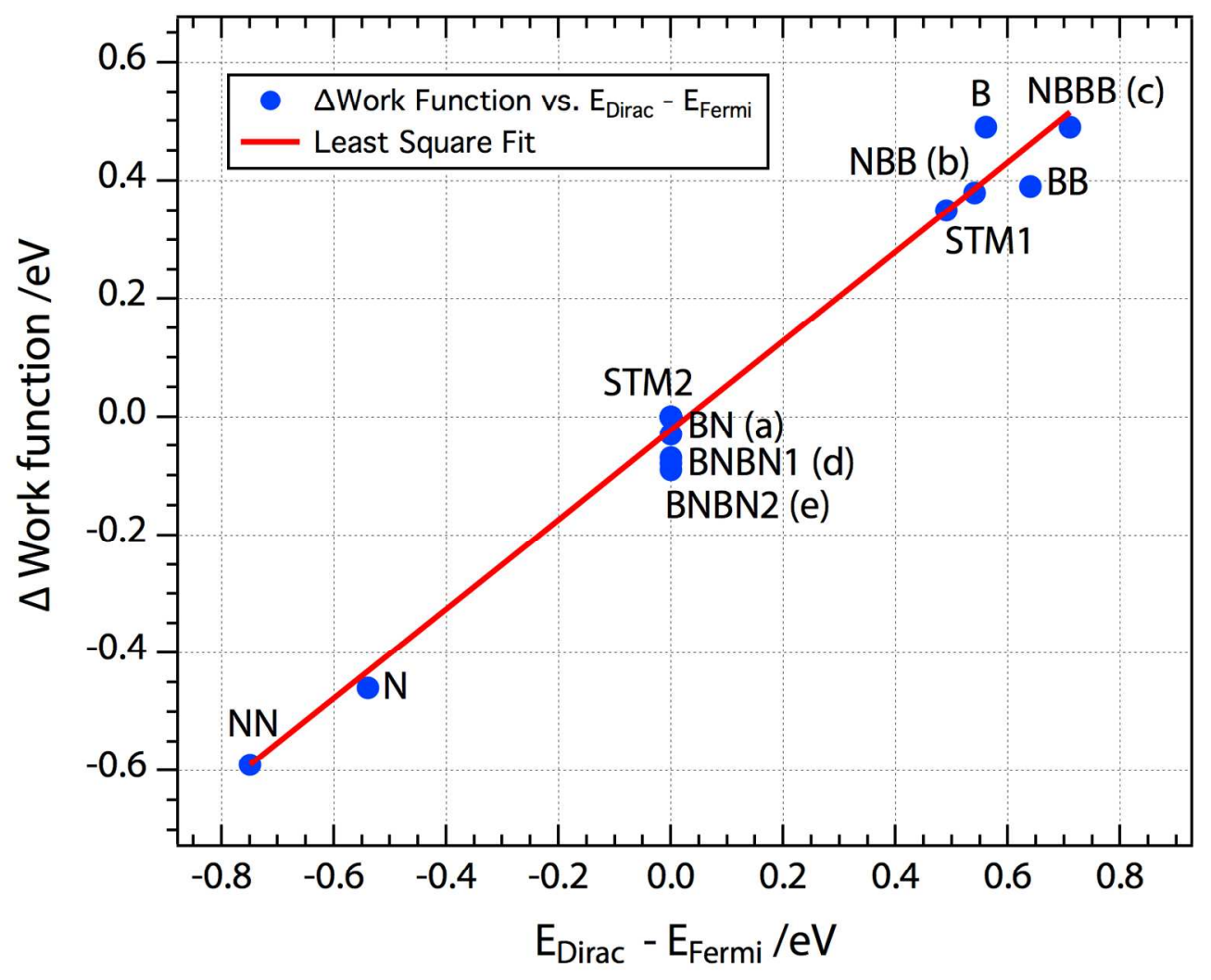

Figure S5. Correlation between computed work function shifts and Dirac point energy shifts for the structures reported in Table 1 of the main text. The linear regression has slope 0.77 and intercept $-0.04 \mathrm{eV}$.

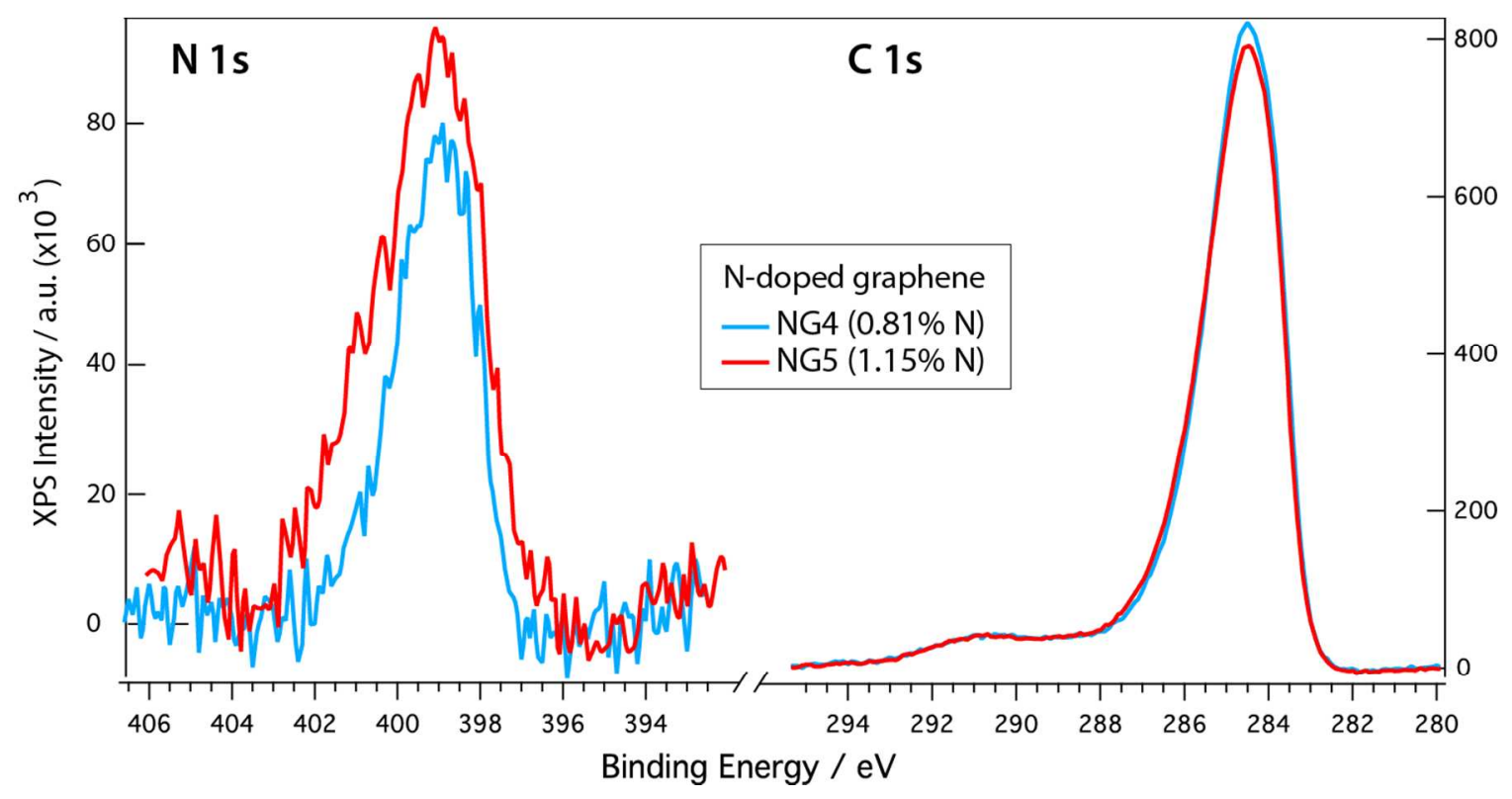

Figure S6. $\mathrm{N} 1 \mathrm{~s}$ and $\mathrm{C} 1 \mathrm{~s}$ XPS data for Nitrogen-doped $\left(\mathrm{NH}_{3}\right.$-grown $\mathrm{CVD}$ ) graphene used to obtain atomic nitrogen concentrations of $1.15 \%$ (NG5) and $0.81 \%$ (NG4). N 1s data was measured at a photon energy of $520 \mathrm{eV}$ and $\mathrm{C} 1 \mathrm{~s}$ data was measured using photon energy of $420 \mathrm{eV}$, so that the $1 \mathrm{~s}$ electrons in both cases are emitted with kinetic energy values in the same range $(\sim 120 \mathrm{eV})$, to obtain an accurate atomic concentration ratio. 


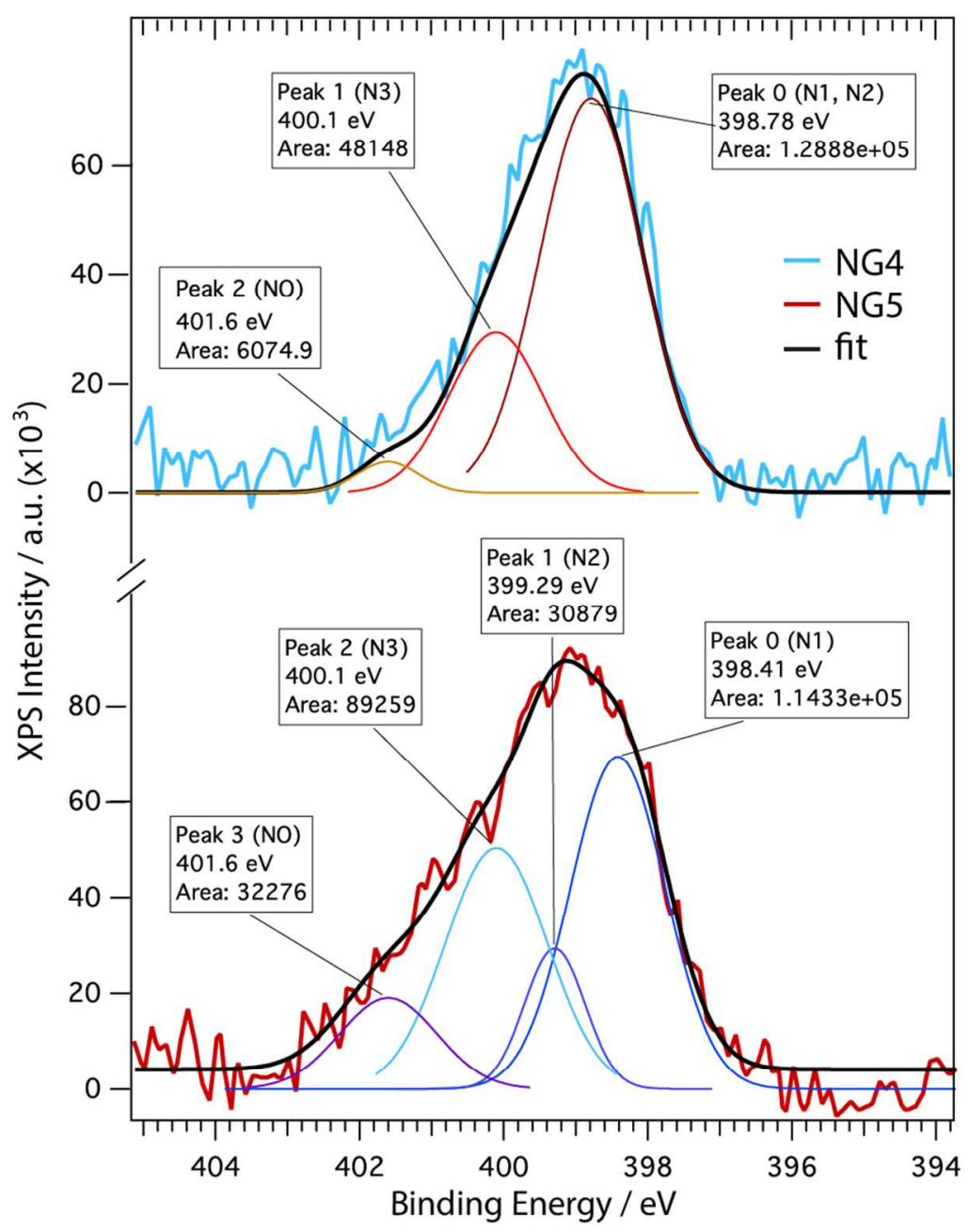

Figure S7. Peak fitting of the experimental N 1s XPS data to identify relative concentrations of different nitrogen species in the $\mathrm{N}$-doped graphene films shown in Fig. S3. The higher concentration of nitrilic $(\mathrm{N}$ bound to one carbon atom (N1) and pyridinic (N bound to two carbon atoms (N2) as compared to graphitic nitrogen (3 carbon bonds (N3)) results in p-type graphene, as discussed in detail in ref. 22 of main text. Details of the peak fitting, summarized in Table S1 are used to correlate doping level with work function shifts based on measurements of p-type graphene shown in Fig. 5 of the main text.

Table S1. Atomic concentration of N-dopants in different forms (N1, N2, N3 and NO) based on XPS data (Figs. S6-S7). N1 (nitrilic) and N2 (pyridinic) dopants have a p-doping effect in graphene while N3 (graphitic) dopants n-dope graphene. The doping effect of surface nitrogen oxide (NO) is unknown. Concentrations are used to correlate doping effect (number of p-type $\mathrm{N}$-dopants minus number of $\mathrm{n}$-type $\mathrm{N}$ dopants, $\mathrm{N}_{\mathrm{p}}-\mathrm{N}_{\mathrm{n}}$, obtained as $\left.(\mathrm{N} 1+\mathrm{N} 2)-\mathrm{N} 3\right)$ with work function change $(\Delta \mathrm{W} . \mathrm{F}$.) in graphene. $\mathrm{N} 1$ and $\mathrm{N} 2$ type dopants have binding energy ranges in close proximity; both types are included in peak 0 in the fit of N 1s XPS for NG4 (Fig. S7).

\begin{tabular}{|c|c|c|c|c|c|c|c|}
\hline & Total N & N1 & N2 & N3 & NO & $\mathbf{N}_{\mathbf{p}}-\mathbf{N}_{\mathbf{n}}(\mathbf{( N 1 + N 2 ) - N 3 )}$ & $\boldsymbol{\Delta}$ W.F. \\
\hline NG4 & $0.81 \%$ & \multicolumn{2}{|c|}{$0.57 \%$} & $0.21 \%$ & $0.03 \%$ & 0.36 & $0.4 \mathrm{eV}$ \\
\hline NG5 & $1.15 \%$ & $0.49 \%$ & $0.13 \%$ & $0.39 \%$ & $0.14 \%$ & 0.23 & $0.2 \mathrm{eV}$ \\
\hline
\end{tabular}




\section{References}

1. Momma, K.; Izumi, F. VESTA 3 for Three-Dimensional Visualization of Crystal, Volumetric and Morphology data. J. Appl. Crystallogr. 2011, 44, 1272-1276. 